\title{
Comparative proteogenomic analysis of the Leptospira interrogans virulence-attenuated strain IPAV against the pathogenic strain 56601
}

Yi Zhong ${ }^{1,2,{ }^{*}, \text { Xiao Chang }}{ }^{3,4, *}$, Xing-Jun Cao ${ }^{3, *}$, Yan Zhang ${ }^{1}$, Huajun Zheng, ${ }^{5}$ Yongzhang Zhu ${ }^{1}$, Chengsong Cai ${ }^{1}$, Zelin Cui ${ }^{1}$, Yunyi Zhang ${ }^{2}$, Yuan-Yuan Li $^{3}$, Xiu-Gao Jiang ${ }^{6}$, Guo-Ping Zhao, ${ }^{2,5}$, Shengyue Wang, ${ }^{5}$ Yixue Li $^{3}$, Rong Zeng ${ }^{3}$, Xuan $\mathrm{Li}^{2}$, Xiao-Kui Guo

${ }^{I}$ Department of Medical Microbiology and Parasitology, Institutes of Medical Sciences, Shanghai Jiao Tong University School of Medicine, Shanghai 200025, China; ${ }^{2}$ Key Laboratory of Synthetic Biology, Institute of Plant Physiology and Ecology, Shanghai Institutes for Biological Sciences, Chinese Academy of Sciences, Shanghai 200032, China; ${ }^{3}$ Key Laboratory of Systems Biology, Institute of Biochemistry and Cell Biology, Shanghai Institutes for Biological Sciences, Chinese Academy of Sciences, Shanghai 200031, China; ${ }^{4}$ Graduate School of the Chinese Academy of Sciences, Beijing 100039, China; ${ }^{5}$ Shanghai-MOST Key Laboratory of Health and Disease Genomics, Chinese National Human Genome Center at Shanghai, Shanghai 201203, China; ${ }^{6}$ National Institute for Communicable Disease Control and Prevention, Chinese Center for Disease Control and Prevention, Changping, Beijing 102206, China

The virulence-attenuated Leptospira interrogans serovar Lai strain IPAV was derived by prolonged laboratory passage from a highly virulent ancestral strain isolated in China. We studied the genetic variations of IPAV that render it avirulent via comparative analysis against the pathogenic $L$. interrogans serovar Lai strain 56601 . The complete genome sequence of the IPAV strain was determined and used to compare with, and then rectify and reannotate the genome sequence of strain 56601. Aside from their highly similar genomic structure and gene order, a total of 33 insertions, 53 deletions and 301 single-nucleotide variations (SNVs) were detected throughout the genome of IPAV directly affecting 101 genes, either in their 5' upstream region or within their coding region. Among them, the majority of the 44 functional genes are involved in signal transduction, stress response, transmembrane transport and nitrogen metabolism. Comparative proteomic analysis based on quantitative liquid chromatography (LC)-MS/MS data revealed that among 1627 selected pairs of orthologs, 174 genes in the IPAV strain were upregulated, with enrichment mainly in classes of energy production and lipid metabolism. In contrast, 228 genes in strain 56601 were upregulated, with the majority enriched in the categories of protein translation and DNA replication/repair. The combination of genomic and proteomic approaches illustrated that altered expression or mutations in critical genes, such as those encoding a Ser/Thr kinase, carbon-starvation protein CstA, glutamine synthetase, GTP-binding protein BipA, ribonucleotidediphosphate reductase and phosphate transporter, and alterations in the translational profile of lipoproteins or outer membrane proteins are likely to account for the virulence attenuation in strain IPAV.

Keywords: genome; proteome; Leptospira; virulence; mutation

Cell Research (2011) 21:1210-1229. doi:10.1038/cr.2011.46; published online 22 March 2011

*These three authors contributed equally to the work.

Correspondence: Shengyue Wang ${ }^{\mathrm{a}}$, Yixue Li ${ }^{\mathrm{b}}$, Rong Zeng ${ }^{\mathrm{c}}$, Xuan $\mathrm{Li}^{\mathrm{d}}$,

Xiao-Kui Guo ${ }^{\mathrm{e}}$

${ }^{a}$ E-mail: wangsy@chgc.sh.cn

${ }^{b}$ E-mail: yxli@sibs.ac.cn

${ }^{\mathrm{c} E}$-mail: zr@sibs.ac.cn

${ }^{\mathrm{d} E}$ E-mail: lixuan@sippe.ac.cn

eE-mail: microbiology@sjtu.edu.cn

Received 20 September 2010; revised 8 December 2010; accepted 20 December 2010; published online 22 March 2011

\section{Introduction}

Leptospira belong to spirochetes, a group of bacteria primitive in the evolution of prokaryotes [1]. Leptospires are thin, coiled microbes with a hook at one or both ends. They are highly motile, obligate aerobe organisms that share features of both Gram-positive and Gramnegative bacteria [2]. Leptospirosis, caused by a diver- 
sity of pathogenic species within the genus Leptospira, has emerged as a globally important zoonotic disease [3, 4]. A wide variety of mammalian hosts, such as rodents, horses, cattle and pigs, may serve as reservoirs. Bacteria persistently colonize the proximal renal tubules of carrier animals; and humans and other animals become infected through exposure to infected urine, contact with urinecontaminated soil and water $[3,5]$ or through the host-tohost transmission cycle [6]. Hosts infected by pathogenic Leptospira display diverse manifestations that range from subclinical infection, a mild influenza-like febrile illness, to severe systemic disease with renal and hepatic failure, extensive vasculitis, pulmonary hemorrhage and death.

With the availability of the whole genome sequence of Leptospira interrogans serovar Lai strain 56601, a highly virulent strain isolated in Sichuan province, China, in 1958 [7], functional genomic research has been carried out in recent years [8-10]. However, key factors that contribute to pathogenesis still remain to be systematically elucidated. The L. interrogans serovar Lai strain IPAV was originally isolated from a patient in China, but details of the isolation record were unavailable. After it was transferred to the N H Swellengrebel Laboratory of Tropical Hygiene, the Royal Tropical Institute, Amsterdam, by Hans Korver, this strain lost its virulence through the process of high-frequency passage as tested in the Institute Pasteur after 1987 (Mathieu Picardeau, personal communication). It was confirmed to belong serologically to the Lai serovar of serogroup Icterohemorrhagiae after transfer to the Department of Medical Microbiology and Parasitology, Institutes of Medical Sciences, Shanghai Jiao Tong University School of Medicine, in 2001. Therefore, the currently available IPAV strain is a candidate suitable for comparative analysis against the Lai-type strain 56601, with the aim of understanding the mechanism of pathogenesis and virulence.

In this study, we first confirmed the avirulent characteristics of strain IPAV and then determined its entire genomic sequence using high-throughput pyrophosphate sequencing [11]. In addition, proteomic MS/MS data were integrated into the genome annotation of IPAV and reannotation of strain 56601. Comparative genomic analysis indicated that the two closely related strains shared highly similar genome structure and gene-order features. Therefore, the biased distribution of a limited number of genetic variations, including insertions, deletions and single-nucleotide variations (SNVs), in certain groups of genes may account for the virulence attenuation in IPAV. Quantitative proteomic analysis also revealed a detectable functional bias in proteomic profiles between the two strains cultured under laboratory conditions, which is likely caused by the genetic variations. These findings were further analyzed with a focus on individual genes, which was important for a better understanding of the molecular pathogenic mechanism of leptospirosis.

\section{Results}

The avirulent character of the L. interrogans strain IPAV as tested on experimental animal models

The virulence of the L. interrogans strain 56601 on experimental animal models with a lethal dose infection was described previously $[12,13]$. The avirulence property of the strain IPAV was tested on leptospirosis animal models as a negative control for strain 56601 infection experiments in our recent work [14], where all experimental animals survived intraperitoneal infection with strain IPAV $\left(5 \times 10^{8}\right)$, whereas an approximate $90 \%$ mortality rate was observed with the complete leptospirosis course for the group of animals inoculated with strain $56601\left(5 \times 10^{8}\right)$.

Meanwhile, 6 days post inoculation, sectioned lung, liver and kidney from guinea pigs infected by strains IPAV and 56601 were subjected to histological analysis via hematoxylin and eosin (H\&E) staining (Figure 1). Tissue slices from guinea pigs injected with IPAV did not present conspicuous pathologic changes (Figure 1A). In
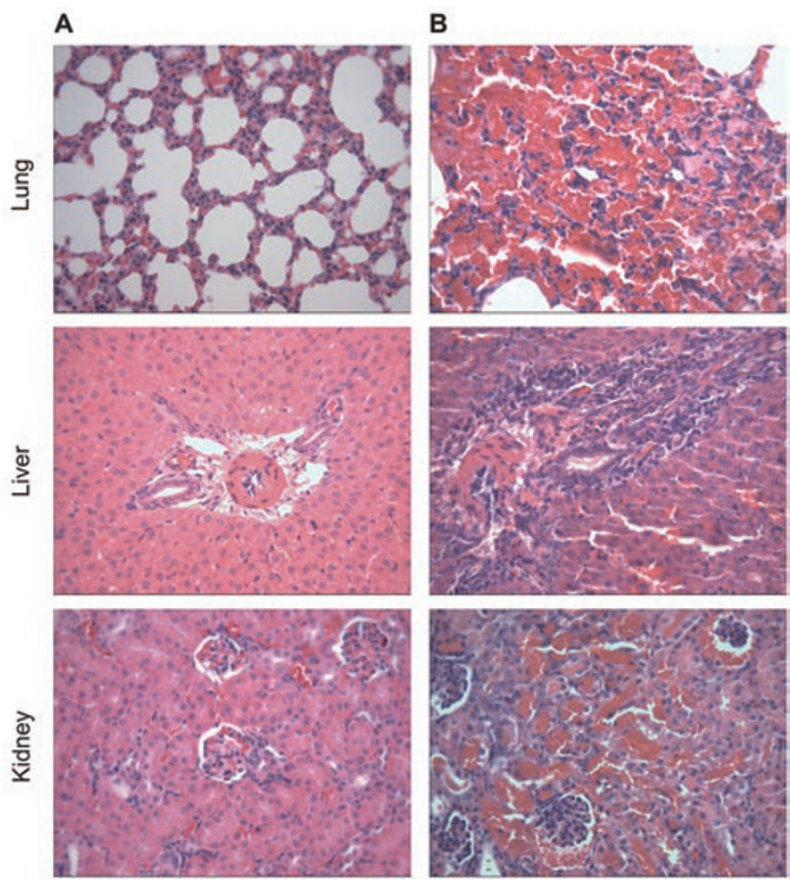

Figure 1 Histological sections in the lung, liver and kidney of guinea pigs infected with $L$. interrogans strain IPAV (A) and strain 56601 (B) (H\&E, magnification $\times$ 400). 
contrast, tissue slices from animals inoculated with strain 56601 produced histopathologic characteristics typical of severe leptospirosis. These included intra-alveolar hemorrhage, edema of alveolar septa and inflammatory infiltrates in the lung; hepatocyte swelling, necrosis, defective hepatic cord formation and increase of monocytes and neutrophils in portal tracts; and glomerular capsules and the lumen of renal tubules pervaded with erythrocytes in kidney (Figure 1B).

General features of the IPAV genome are highly similar to that of strain 56601

The complete genome of $L$. interrogans strain IPAV was sequenced along with the rectification of strain 56601. The genome of strain IPAV consists of two circular chromosomes (Figure 2). The large chromosome (CI) consists of $4349158 \mathrm{bp}$ with an average GC content of $35.02 \%$, which is $10396 \mathrm{bp}$ larger than that of strain 56601. The small chromosome (CII) consists of 359372 bp with an average GC content of $35.14 \%$, identical to that of strain 56601 (Table 1, GenBank accession number CP001221 for CI and CP001222 for CII). The replication origins of both $\mathrm{CI}$ and CII were located by referring to those of strain 56601, originally identified by GC skew analysis [7]. In total, 3726 protein coding sequences (CDSs) were predicted, with biological function assigned for 2320 CDSs. In addition, 1918 CDSs could be placed into clusters of orthologous groups (COGs) [15] via homology search. We identified approximately 56 intact insertion sequence (IS) elements scattered throughout the strain IPAV genome. Meanwhile, we identified 55 IS elements in the rectified genome of strain 56601, almost twice as many as were reported in the previous study (30 ISs) [7]. Most of the ISs are identical between the two strains, except for three in strain IPAV (IS1500, 19089631910197; ISlin1, 3392331-3393734; ISlin1, 35250683526455 ) and two in strain 56601 (ISlin 1, 32255563227302; ISlin1, 3227341-3228607). Compared with Leptospira biflexa, the existence of a large number of ISs suggests that IS integration events happened continuously throughout the history of these strains and that the five different ISs between the two strains might have happened after the two strains diverged.

\section{Rectification of the strain 56601 genomic sequence fol- lowed by reannotation}

Some sequencing errors that existed in the originally published strain 56601 genome were resolved. First, the sequence of an approximately 6.7-kb DNA fragment that was missing in the previous genome was identified and added at revised CI coordinates of 3181009 to 3187 723 , between the originally annotated CDSs of LA_3200
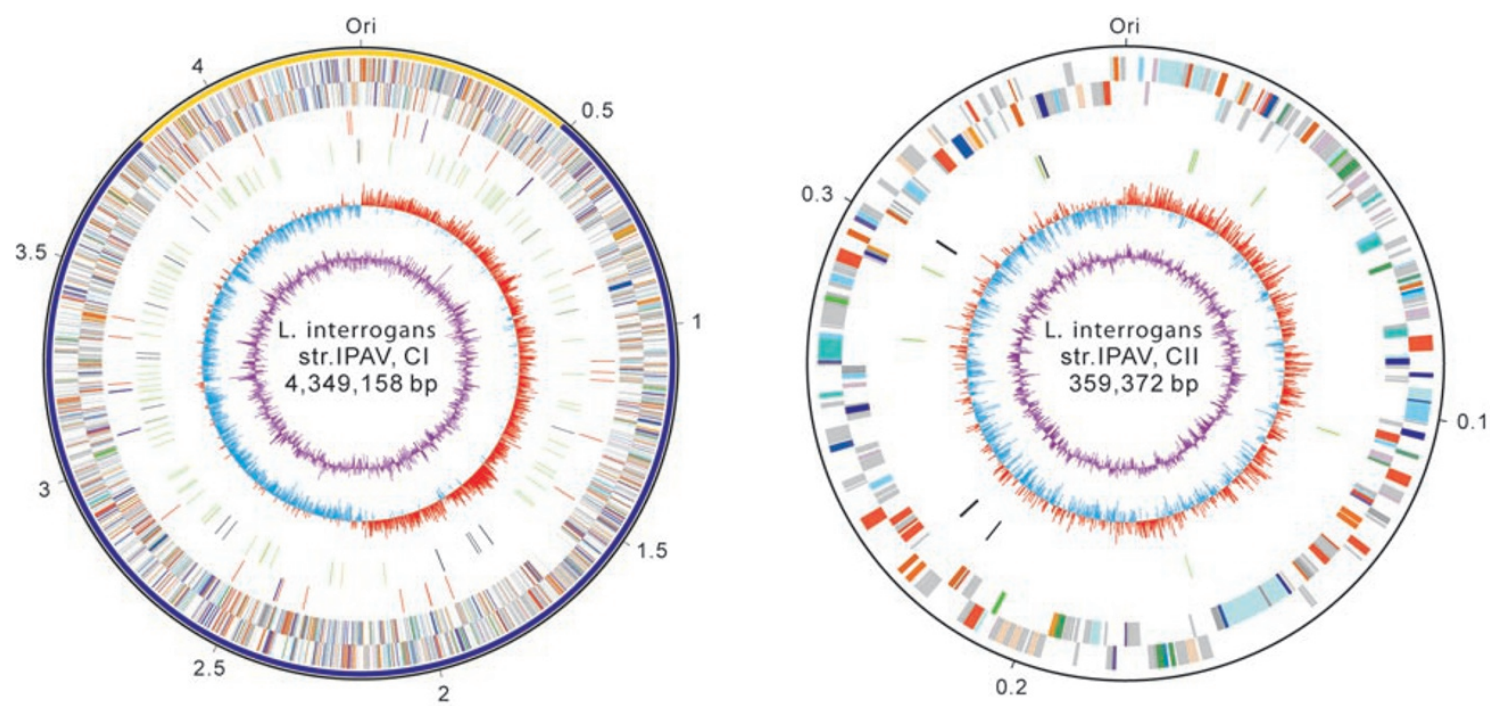

Figure 2 Circular representation of IPAV chromosomes with predicted CDSs. Left, large chromosome (Cl); right, small chromosome (CII). The outer scales are numbered in megabases from the origins of replication (Ori). Circles range from 1 (outer circle) to 7 (inner circle) for $\mathrm{Cl}$ and from I (outer circle) to $\mathrm{V}$ (inner circle) for Cll. Circle 1, representation of genome structure when compared with $L$. interrogans strain Fiocruz L1-130 (yellow, the collinear region; blue, the large inverse region). Circle $2 / I$ and $3 / I I$, genes on plus and minus strands (colors representing functional categories according to COGs). Circle 4, tRNA genes (red) and rRNA genes (blue). Circle 5/III, IS elements (both intact and remnant are included; green, ISlin1; black, other types of ISs). Circle 6/IV, GC skew (calculated using a 2500 bp (Cl)/500 bp (ClI) window sliding 200 bp (Cl)/100 bp (CII) at a time; red, values > 0; green, values $<0)$. Circle $7 / \mathrm{N}, \mathrm{GC}$ content. 


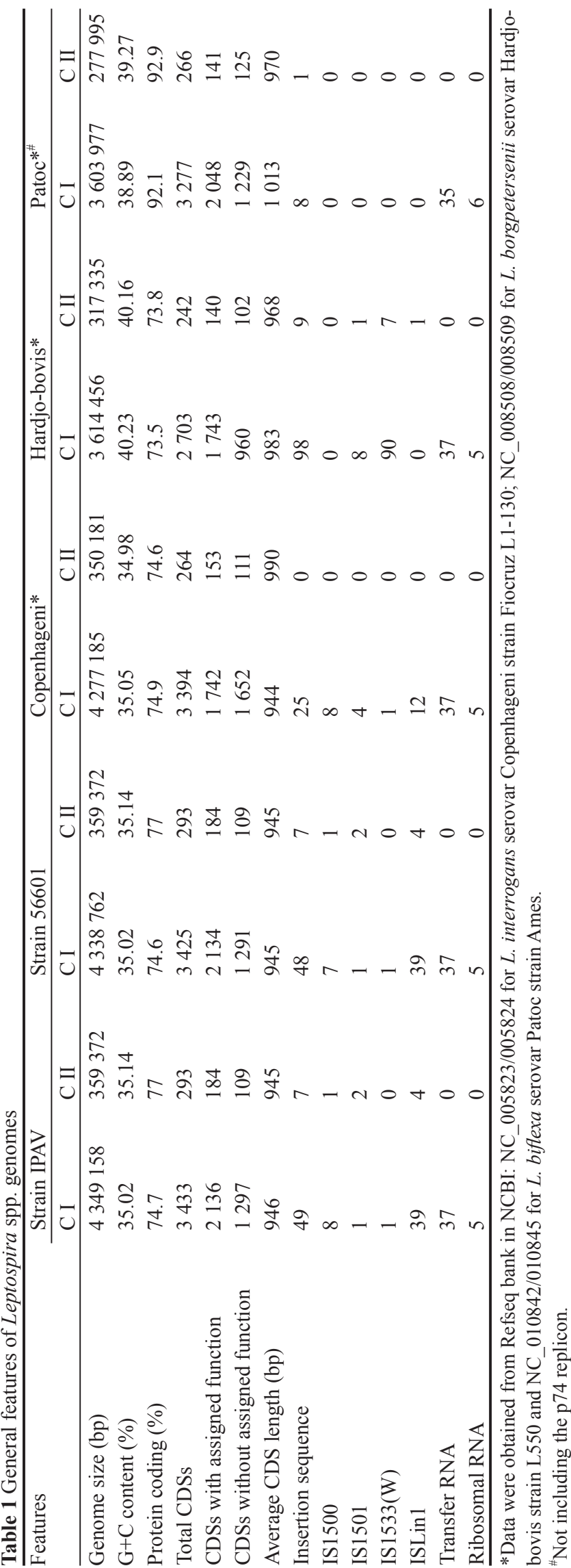

and LA_3201. This region encodes a 23S ribosomal RNA $(r r l)$, a putative lipoprotein that was validated by mass spectrometry (MS), and the N-terminal 89 aa of LA 3201, which increased its CDS to 250 aa. Second, the sequence of an approximately 6.5-kb DNA region spanning the revised CI coordinates 3248961 to 3255 503 was erroneously reversed in the originally published Lai 56601 genome map. Four other large fragments were rectified, including sequence rectification from 414967 to 415363 (LA 0417), a sequence addition from 2051 058 to 2051577 (LA_2081a and LA_2083) with respect to the revised CI coordinates, a sequence addition from 332934 to 333358 (LB_340 and LB_340a) with respect to the revised CII coordinates, and a deletion of an 831bp direct repeat flanking the Genome Island I (GI I) [16]. Finally, 43 single nucleotides were rectified according to the resequencing of the corresponding sites. The updated nucleotide sequence for strain 56601 contains 4698134 bp, of which CI contains 4338762 bp and CII contains 359372 bp.

The completely reannotated genome of the virulent L. interrogans serovar Lai strain 56601 was deposited in the NCBI database with the original accession numbers (AE010300 for CI and AE010301 for CII). Among the predicted 4727 CDSs annotated previously [7], onefifth of them were shorter than 70 codons, mainly due to the short (30 codons) cutoff value. These "hypothetical proteins" shared no homology with known proteins/domains and are not expressed in the cell, as we discovered through proteomic profiling. In this reannotation, the length of coding sequences was reevaluated according to our new proteomic knowledge [17]. With the length of 60 codons as the cutoff value, only 3653 CDSs were predicted for strain 56601 . Then, 65 CDSs shorter than 60 codons were added manually based on the relatively high confidence predictions. This reannotated genome of strain 56601 contains $3718 \mathrm{CDSs}$, with 1088 being removed from the previous version and 79 being newly included. Although 18 of the newly added CDSs have assigned functions, 6 are truncated transposases, and the remaining proteins are "hypothetical". The original LA designations for the CDSs annotated in the preceding genome database were maintained. The additional or revised CDSs annotated by this work were designated by appending their $5^{\prime} \mathrm{CDSs}$ with a lowercase alphabetic letter (e.g., LA_0395a following LA_0395, and LA_0727a and LA 0727b following LA 0727). All 3718 CDSs were checked for their probable functions, which resulted in 2 318 CDSs with assigned biological functions and 148 conserved hypothetical proteins similar to the proteins in the NCBI's CDD database. The remaining 1252 hypothetical proteins do not have enough homology with proteins of 
other genera and are unique to the Leptospira genome.

\section{Proteomics-assisted genome annotation}

To improve the annotation of the genome of $L$. interrogans, MS-based proteomic analysis was performed for both strains grown under routine experimental conditions. Using MS/MS spectra obtained from strains 56601 and IPAV, sequences were searched against 22216 (strain 56601 ) and 22269 (strain IPAV) six-frame translated proteins that served as theoretical databases. We found additional 13 genes that were missed in the previous gene prediction process employing computational software. A detailed analysis indicated that among the 13 missed genes, 8 overlapped with their adjacent genes on one end, 2 were located completely inside other genes, and the remaining 3 are in the intergenic regions. Of these 13 CDSs, the smallest one is a hypothetical protein (67 aa) in IPAV. For the two missed genes located completely inside other genes (LIF_A2691 (3 323 647-3 323 850) inside LIF_A2690 (3 323 351-3 324 025); LA_0853a (852 272-852 679) inside both LA 0853 (851 443-852 315) and LA_0854 (852 312-853 562)), we did not remove the corresponding CDSs that conflicted with the two proteomic-profiling-verified genes in terms of translational frame because we indeed detected proteomic expression of both frames of overlapping genes in the two strains.

Using MS/MS spectra to search the CDS databases, a total of 2608 and $2673 \mathrm{CDSs}$ were detected in strains IPAV and 56601, respectively. Because the predicted proteins aligned to at least two unique peptides from the MS data, they were considered reliably validated. Further functional analysis focused on the $2078(55.8 \%)$ and 2 225 (59.8\%) CDSs confirmed with at least two unique peptide hits from IPAV and 56601, respectively (Figure 3). We evaluated these proteins based on their COG functional category classification. Of the 1918 proteins from IPAV with assigned COG categories, $70.8 \%$ were detected in proteomic profiling, whereas only $39.8 \%$ of the remaining 1808 CDSs were confirmed. Similarly, in strain $56601,74.8 \%$ of the 1918 proteins with assigned COG categories were detected and only $43.9 \%$ of the remaining $1800 \mathrm{CDSs}$ were observed. Similar statistics were also observed in the proteomic profiling of Mycoplasma pneumoniae and Mycoplasma mobile [18, 19]. We also compared our data for both IPAV and 56601 with the recently published proteome-wide cellular protein data for the L. interrogans serovar Copenhageni [20]. A total of 1703 orthologous proteins $(72.1 \%$ of all proteins identified in IPAV and 56601) were shared by the two studies, including 1233 functional proteins and 366 hypothetical

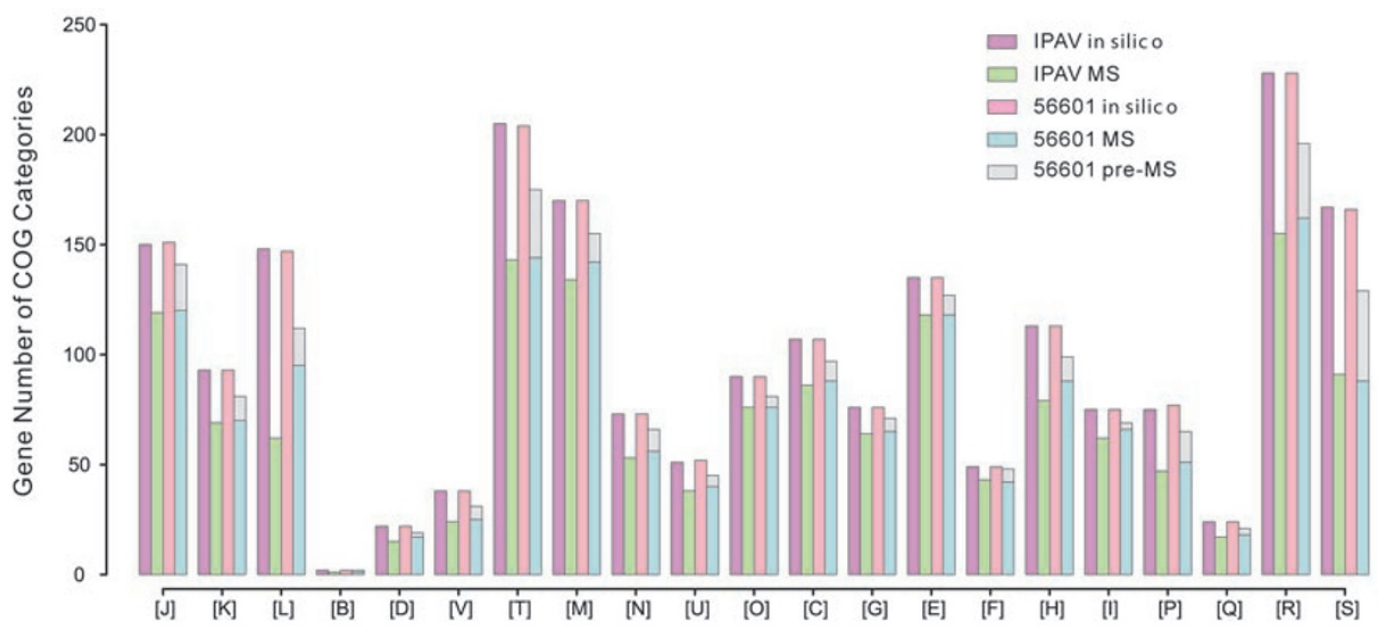

Figure 3 Functional category of in silico predicted proteins and MS-validated proteins. The colors used in the bars represent different objects: purple, IPAV all predicted proteins; green, IPAV MS-validated proteins; red, 56601 all predicted proteins; blue, 56601 MS-validated proteins; gray, 56601 proteins that were not detected in this work but confirmed by our previous MS data. Proteins were clustered by COG assignment: $(\mathrm{J})$ translation, ribosomal structure and biogenesis; (K) transcription; $(\mathrm{L})$ replication, recombination and repair; (B) chromatin structure and dynamics; (D) cell-cycle control, cell division and chromosome partitioning; (V) defense mechanisms; ( $\mathrm{T}$ ) signal transduction mechanisms; $(\mathrm{M})$ cell wall/membrane/envelope biogenesis; (N) cell motility; (U) intracellular trafficking, secretion and vesicular transport; $(\mathrm{O})$ posttranslational modification, protein turnover and chaperones; (C) energy production and conversion; $(\mathrm{G})$ carbohydrate transport and metabolism; (E) aminoacid transport and metabolism; $(\mathrm{F})$ nucleotide transport and metabolism; $(\mathrm{H})$ coenzyme transport and metabolism; (I) lipid transport and metabolism; $(P)$ inorganic ion transport and metabolism; $(\mathrm{Q})$ secondary metabolites biosynthesis, transport and catabolism; (R) general function prediction only; (S) function unknown. 
proteins.

IS elements as markers for phylogenetic trace of Leptospira species and subspecies

IS elements usually contribute significantly to the plasticity of genomic structure. We examined the differences of the IS elements by their types and numbers among the seven sequenced leptospiral genomes and noticed that some of them might serve as molecular markers to trace the divergence of distinct strains from their common progenitor (Supplementary information, Table $\mathrm{S} 1)$.

Despite a wide range of differences in the structural arrangement of the genome, all strains of $L$. interrogans shared a 90-bp ISlin1 remnant at the same site of their CI chromosomes (coordinates 1388225 to 1388314 as for L. interrogans IPAV) with that of Leptospira borgpetersenii (coordinates 2651714 to 2651804 as for $L$. borgpetersenii JB109, $85 \%$ identical to that of IPAV) (Figure 4, Supplementary information, Table S1). This is the only IS marker that is shared by all pathogenic Leptospira species known so far, which obviously indicates that $L$. interrogans and $L$. borgpetersenii evolved from a common ancestor after the integration event of ISlin1 at this site. Similarly, various types of IS elements at common loci can be observed within strains of the same species, for example, the two strains of $L$. biflexa, the two strains of $L$. borgpetersenii, the three strains of $L$. interrogans and the two strains of $L$. interrogans serovar Lai (Figure 4, Supplementary information, Table S1). However, there is no single common IS element that is shared among all seven strains, which seems mainly due to the fact that few IS elements reside in the two saprophytic species $L$. biflexa (six or nine copies each, respectively). This result suggests that IS-related variation in the process of evolution of Leptospira occurred mainly in the pathogenic species that diverged from a common progenitor with the saprophytic Leptospira.

The distribution of different IS elements differs dramatically among Leptospira species [6, 21]. We constructed the phylogenetic tree for ISlin1, the dominant and most polymorphic mobile element in L. interrogans (Supplementary information, Figure S1). ISlin1 on the large chromosome was selected when there was greater than $80 \%$ sequence identity and reciprocal length coverage in both. In IPAV, the ISlin1 can be grouped into two clusters, groups I and II. Group I includes nine ISs, six of which have cognates in serovar Copenhageni. The ISlin1 F29 in IPAV is the cognate of ISlin1 C3 in Copenhageni, and both are located at the bottom of the trees and have low similarity with other ISlin 1 in IPAV and Copenhageni, indicating a distinct history in the ances-

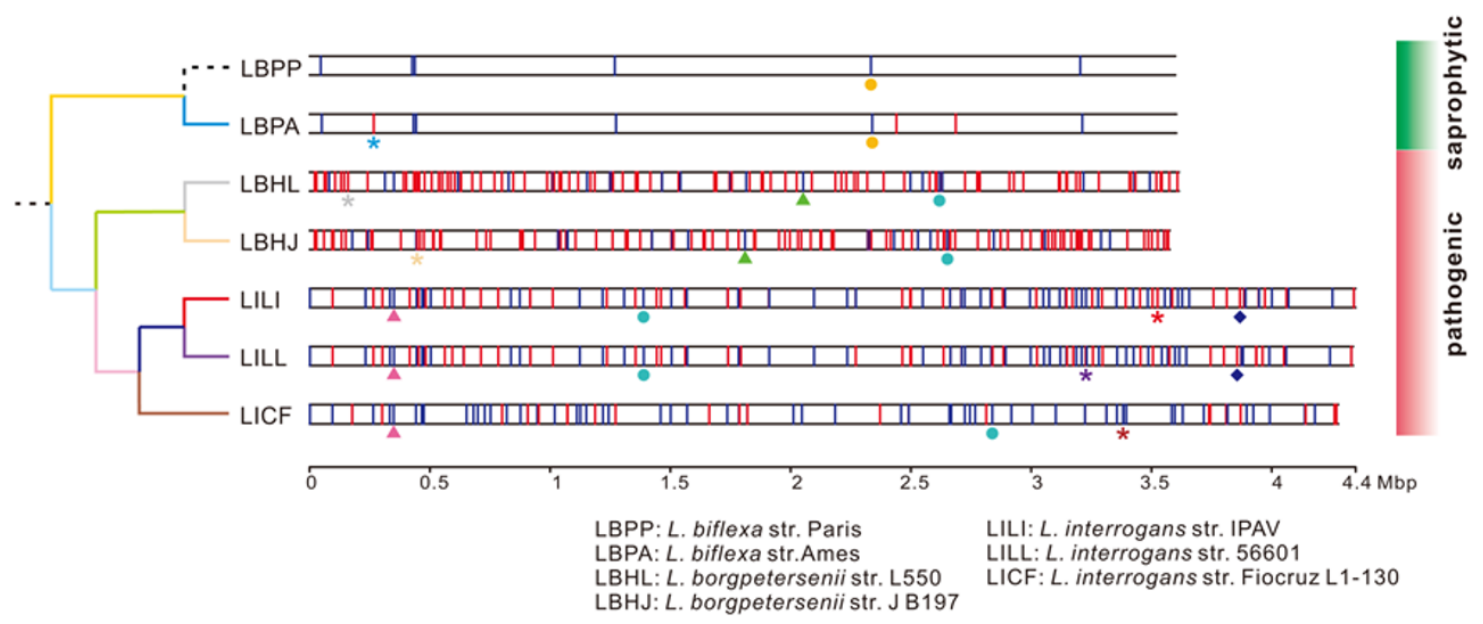

Figure 4 Distribution of IS elements in large chromosomes of leptospires. The scale is numbered in megabases from the origins of replication. The bars represent the chromosomes. The short vertical lines in each bar indicate the IS elements at different coordinates (red, intact ISs, >1 000 bp; blue, remnant ISs, 100-1 000 bp). The symbols below the genomes are selected IS markers (pathogenic Leptospira specific, sky blue circles; saprophytic Leptospira specific, orange circles; L. borgpetersenii specific, green triangles; $L$. interrogans specific, magenta triangles; $L$. interrogans serovar Lai specific, navy blue rhombuses; strain Fiocruz L1-130 specific, brown asterisk; strain 56601 specific, purple asterisk; strain IPAV specific, red asterisk; strain JB197 specific, light yellow asterisk; strain L550 specific, gray asterisk; strain Ames specific, blue asterisk). For the IS markers in each evolutionary level, only one was selected to illustrate in this figure. The colors of the branches in the phylogenic tree consistent with the colors used on the IS markers and the dashed lines indicate that no IS marker is identified at the corresponding levels. 
tor of serovars Lai and Copenhageni. In group II, 30 ISs are clustered together in a large clade. Because they are highly similar to each other, this group is not well separated by bootstrap analysis. Most members of this group have no cognate in Copenhageni and are intact from the beginning to the end in each copy. This result strongly suggests that ISs in this group still have mobile activities that could lead to frequent insertion and recombination events such as the large inversion observed between serovars Lai and Copenhageni, which is suggested to be caused by ISlin1 F33 and ISlin1 F6 [21]. In conclusion, although Copenhageni harbors 12 seemingly intact ISlin1 elements, at least 10 of them have lost their transposon function due to truncation, and according to Johan Malmstron [20], no ISlin1 transposase was detected by MS in Copenhageni. Most of the ISlin1 elements in IPAV or 56601 may still retain their mobile activity, especially those of group II, and many members seem to have been inserted into the host chromosome after serovar divergence.

Comparative genomic analysis of $L$. interrogans strains IPAV and 56601

A comparative genomic study of the two serovar Lai strains, the avirulent IPAV and the highly virulent type 56601 strain, was performed with the genome sequence of serovar Copenhageni as an outlier to distinguish strain-specific variations. Comprehensive genetic conservation was observed from chromosome-by-chromosome alignments between the two serovar Lai strains, with a total of 33 insertions and 53 deletions detected in IPAV relative to 56601 (Supplementary information, Table S2). However, only nine $(27.3 \%)$ insertions are $>1$-bp variations, whereas $33(62.3 \%)$ deletions are $>1$-bp variations. In addition, $301 \mathrm{SNVs}$ and 3 large-sequence polymorphisms (LSPs) were identified in either the CDSs or the intergenic regions (Supplementary information, Table S3). Among these variations, a total of 101 genes, including 44 functional genes (Table 2), 29 hypothetical genes and 28 transposases, were affected either in the coding region or in the $5^{\prime}$ upstream region. Furthermore, by comparing the genetic variations in the 44 functional genes with their corresponding loci in L. interrogans serovar Copenhageni, 18 gene variations were designated "56601-specific" because these sequences in IPAV are identical to that of serovar Copenhageni. Variations in the remaining 26 genes were consequently termed "IPAVspecific".

The largest insertion (14 $025 \mathrm{bp}$ ) in the genome of IPAV was found between LIF A2852a and LIF A2862, from 3516796 to 3530821 (Figure 5A). It introduced nine CDSs, including genes encoding a putative lipopro- tein (LIF_A2853), an RNA polymerase ECF-type sigma factor (LIF A2854), an EAL domain-containing protein (LIF A2857, similar to diguanylate phosphodiesterase), and five hypothetical proteins (LIF_A2855, LIF_A2856, LIF_A2858, LIF_A2860 and LIF_A2861) as well as an ISlin1 transposase (LIF A2859). Although our proteomic study identified four proteins (LIF_A2854, LIF_A2855, LIF_A2857 and LIF_A2858), only LIF_A2858 is unique in the genome. The other three have their paralogous genes elsewhere. This 14-kb DNA fragment was also present with a high degree of similarity in the pathogenic serovar Copenhageni strain, only missing the ISlin1. In reference to our analysis of the IS markers, this intact mobile element (ISlin1 F31) is a member of the group II ISs (Supplementary information, Figure S1A). We infer that the 14-kb DNA fragment should exist in the ancestral of L. interrogans serovar Lai IPAV and 56601 and that the ISlin1 F31 was integrated before the ancestor diverged into different lineages. In one of these lineages, this region was deleted via ISlin1-induced DNA translocation, as was observed in 56601.

Another insertion in IPAV occurred in the Type I restriction modification $(\mathrm{R} / \mathrm{M})$ gene cluster (Figure $5 \mathrm{~B}$ ). This defense mechanism system consists of HsdM, HsdS, HsdR and sometimes a tRNA ${ }^{\text {Lys }}$-specific anticodon nuclease (ACNase), which, in combination, carries out DNA methylation and restriction functions [22]. In the IPAV strain, we identified the intact Type I R/M gene cluster with an anticodon nuclease gene incorporated (LIF A2572-LIF A2575). In strain 56601, however, the anticodon gene LA 3199 and its downstream gene $h s d R$ (LA 3200) are truncated as a result of a $2796-\mathrm{bp}$ deletion. In fact, MS data revealed that HsdM and HsdS had peptide hits in both strains, but HsdR, responsible for the endonuclease activity, was detected only in IPAV. Because the homologous region in Copenhageni exhibits high similarity to that of the IPAV strain, the corresponding nucleotides in strain 56601 were likely lost after the segregation of the two strains. One may conclude that the DNA cleavage and tRNA recognition mechanisms in strain 56601 are impaired.

Other large ( $>40 \mathrm{bp})$ insertions/deletions in strain IPAV include tandem repeats and gains or losses of nucleotides in either CDSs or intergenic regions. Insertions in CDSs include two hypothetical proteins (LIF A1368 and LIF_A3272) and four transposases (LIF A1558, LIF A1559, LIF A2750 and LIF A2751). It is worthwhile to mention that LIF_A1368 is a gene located at the end of the lipopolysaccharide (LPS) biosynthetic locus. We did not detect its translation product by MS analysis. If it is involved in LPS biosynthesis, further study is necessary because mutations in this locus have 
Table 2 Genes that have genetic variations in the two strains

\begin{tabular}{|c|c|c|c|c|c|}
\hline$\overline{\text { IPAV }^{a}}$ & Variation $^{\mathrm{b}}$ & $56601^{\mathrm{a}}$ & $\mathrm{Spec}^{\mathrm{c}}$ & Gene product & rot Quant Alter \\
\hline LIF_A0265 & snv (n) & LA_0312 & IPAV & Cell wall hydrolase & $*$ \\
\hline LIF_A1206 & $\operatorname{snv}(s)$ & LA_1499 & IPAV & Cytoplasmic membrane protein & $*$ \\
\hline LIF_A1400 & del & LA_1731 & IPAV & Biotin/lipoate protein ligase & $*$ \\
\hline LIF_A2549 & del (up) & LA_3166 & IPAV & Metallophosphoesterase & $*$ \\
\hline LIF_B181 & snv (s) & LB_225 & IPAV & Lipoprotein & $*$ \\
\hline LIF_B239 & $\operatorname{snv}(\mathrm{n})$ & LB_298 & IPAV & L-lysine 2, 3-aminomutase & $*$ \\
\hline LIF_A0087 & $\operatorname{snv}(\mathrm{n})$ & LA_0098 & 56601 & tRNA (Uracil-5-)-methyltransferase TrmA & * \\
\hline LIF_A2574 & insert & LA_3199 & 56601 & Anticodon nuclease prrC & * \\
\hline LIF_A2853 & insert & - & 56601 & Putative lipoprotein & * \\
\hline LIF_A0252 & del & LA_0299 & IPAV & Carbon-starvation protein A, CstA & $\mathrm{N}$ \\
\hline LIF_A1136 & $\operatorname{snv}(\mathrm{n})$ & LA_1422 & IPAV & Serine/threonine kinase with GAF and PP2C domains & $\mathrm{N}$ \\
\hline LIF_A1290 & $\operatorname{snv}(n)$ & LA_1603 & IPAV & Glycosyl transferase & $\mathrm{N}$ \\
\hline LIF_A1058 & $\operatorname{snv}(\mathrm{n})$ & LA_1313 & IPAV & Glutamine synthetase GlnA & -- \\
\hline LIF_A1137 & del & LA_1422 & IPAV & Serine/threonine kinase with GAF and PP2C domains & -- \\
\hline LIF_A2090 & snv (n) & LA_2554 & IPAV & Phosphate sodium symporter & -- \\
\hline LIF_A3093 & snv (n) & LA_3871 & IPAV & Acriflavine resistance protein & - \\
\hline LIF_A1928 & snv (n) & LA_2360 & 56601 & Ribonucleotide reductase NrdA & -- \\
\hline LIF_A0023 & snv (n) & LA_0025 & IPAV & Flagellar switch complex protein, FliG & $=$ \\
\hline LIF_A0264 & snv (n) & LA_0311 & IPAV & Metal-dependent hydrolase & $=$ \\
\hline LIF_A0333 & $\operatorname{snv}(n)$ & LA_0391 & IPAV & ATP-dependent protease ClpA & $=$ \\
\hline LIF_A0845 & $\operatorname{snv}(n)$ & LA_1045 & IPAV & Membrane carboxypeptidase & $=$ \\
\hline LIF_A0876 & snv (n) & LA_1082 & IPAV & ADP-ribose pyrophosphatase & $=$ \\
\hline LIF_A1181 & snv (n) & LA_1471 & IPAV & Memb-bound $\mathrm{H}^{+}$translocate pyrophosphatase $\left(\mathrm{H}^{+}\right.$-PPase $)$ & $=$ \\
\hline LIF_A2430 & $\operatorname{snv}(\mathrm{s})$ & LA_2989 & IPAV & $\mathrm{NH}(3)$-dependent $\mathrm{NAD}(+)$ synthetase & $=$ \\
\hline LIF_A2439 & $\operatorname{snv}(\mathrm{s})$ & LA_3002 & IPAV & tRNA nucleotidyltransferase/poly A polymerase & $=$ \\
\hline LIF_A2491 & $\operatorname{snv}(n)$ & LA_3085 & IPAV & Guanosine polyPi pyrophosphohydrolase/synthetase SpoT & $=$ \\
\hline LIF_A3424 & snv (n) & LA_4291 & IPAV & Putative lipoprotein & $=$ \\
\hline LIF_A0210 & $\operatorname{snv}(n)$ & LA_0243 & 56601 & Cytochrome c oxidase polypeptide I CyoB & $=$ \\
\hline LIF_A0392 & del & LA_0459 & 56601 & $\mathrm{~N}$-acetylmuramoyl-L-alanine amidase & $=$ \\
\hline LIF_A1716 & $\operatorname{snv}(\mathrm{s})$ & LA_2114 & 56601 & ATP-binding protein - ABC transporter complex & $=$ \\
\hline LIF_A2120 & insert & LA_2591 & 56601 & Flagellar MS-ring protein FliF & $=$ \\
\hline LIF_A3034 & snv (up) & LA_3793 & 56601 & Putative hemolysin & $=$ \\
\hline LIF_A3075 & del & LA_3845 & 56601 & AcrR family transcriptional regulator & $=$ \\
\hline LIF_A0628 & $\operatorname{snv}(\mathrm{n})$ & LA_0765 & IPAV & RNA polymerase alpha subunit & + \\
\hline LIF_A1885 & $\operatorname{snv}(n)$ & LA_2309 & IPAV & Long-chain-fatty-acid CoA ligase & ++ \\
\hline LIF_A1107 & $\operatorname{snv}(n)$ & LA_1378 & 56601 & GTP-binding protein BipA & ++ \\
\hline LIF_A2500 & snv (n) & LA_3096 & 56601 & Anti-sigma factor antagonist & ++ \\
\hline LIF_A2698 & $\operatorname{snv}(n)$ & LA_3365 & 56601 & Hydroxyethylthiazole kinase ThiM & ++ \\
\hline LIF_A0838 & snv (n) & LA_1036 & IPAV & His kinase and response regulator hybrid protein & $\mathrm{Y}$ \\
\hline LIF_A0515 & del & LA_0632 & 56601 & PEP-dependent enzyme IIA component of the PTS system PtsN & $\mathrm{Y}$ \\
\hline LIF_A1585 & del & LA_1963a & 56601 & Multidrug efflux pump & $\mathrm{Y}$ \\
\hline LIF_A2575 & insert & LA_3200 & 56601 & Type I restriction enzyme hsdR & Y \\
\hline LIF_A2854 & insert & - & 56601 & RNA polymerase ECF-type sigma factor & Y \\
\hline LIF_A2857 & insert & - & 56601 & Signal transduct protein containing EAL domain & Y \\
\hline
\end{tabular}

${ }^{a}$ The transposases and hypothetical proteins in both strains are not included.

${ }^{\mathrm{b}} \mathrm{Snv}$ (n), nonsynonymous SNV in coding region; snv (s), synonymous SNV in coding region; snv (up), SNV in gene upstream; del, nucleotide deletion in coding region; del (up), nucleotide deletion in gene upstream; insert, nucleotide insertion in coding region.

${ }^{\mathrm{c}} \mathrm{Specificity}$ of each variation is designed by checking its corresponding site on $L$. interrogans serovar Copenhageni.

$\mathrm{d}_{++}$, the protein abundance of IPAV is significantly higher than that of strain $56601(P<0.01)$; + , higher $(P<0.05)$; Y, the protein is only expressed in IPAV; --, the protein abundance of IPAV is significantly lower than that of strain $56601(P<0.01)$; - , lower $(P<0.05)$; N, the protein is only expressed in strain $56601 ;=$, the protein abundance in each strain has no significant difference. ${ }^{*}$, the protein is not expressed in either strain. 


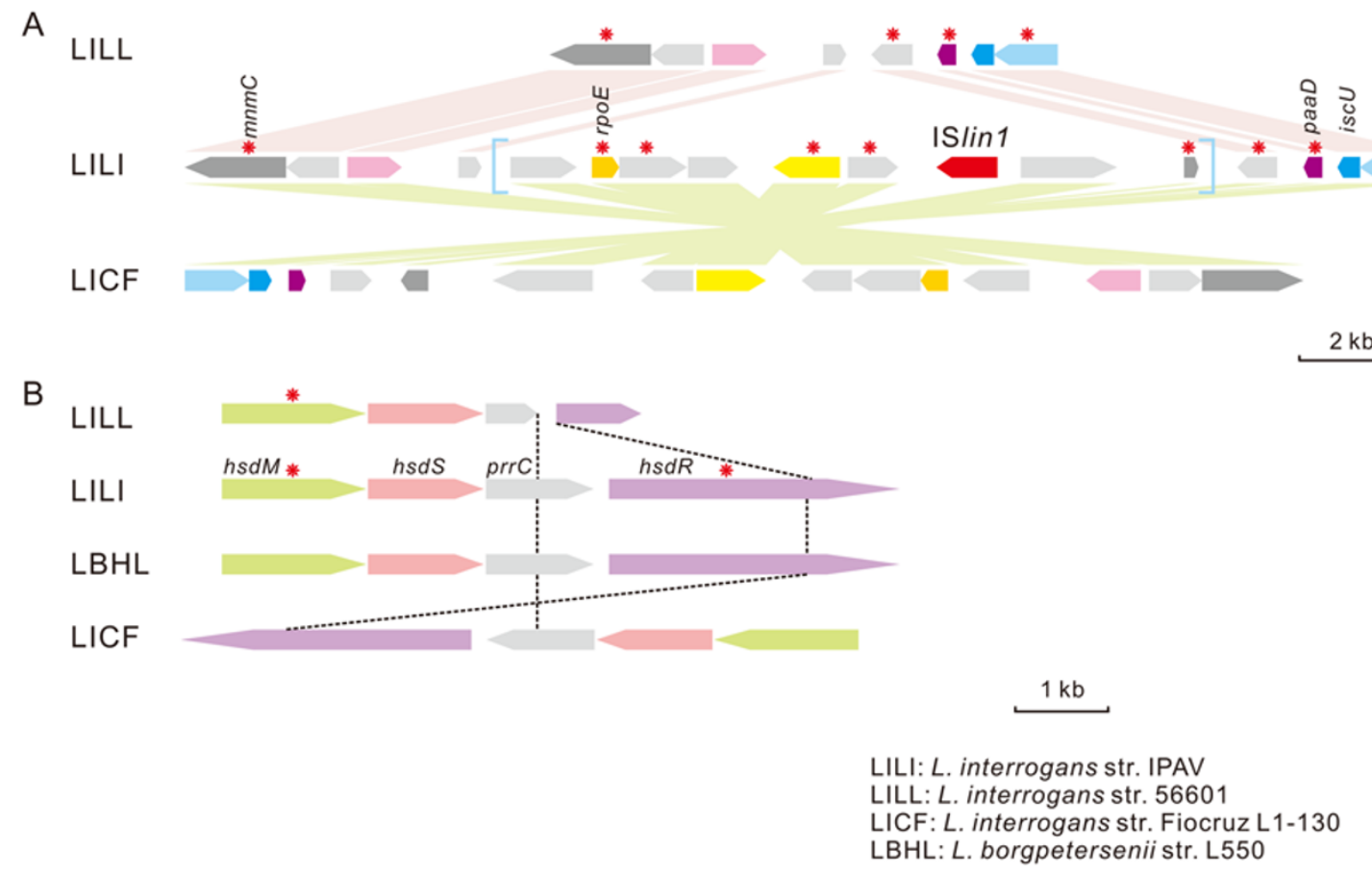

Figure 5 Large Indels in the genomes of $L$. interrogans strains IPAV or 56601. (A) Loss of 14-kb DNA sequence in strain 56601. (B) Type I R/M gene cluster. Genes are color coded according to their functional categories. A red asterisk is labeled above a gene if its product can be detected by MS.

been demonstrated to strongly affect the virulence [23]. Deletions in IPAV are equivalent to insertions in strain 56601, which include six hypothetical proteins (LA_0509, LA_1665, LA_1761, LA_1762, LA_2773 and LA_3161) and four transposases (LA_3249, LA_3250, LA_3252 and LA_3253).

Protein quantification and comparative proteomic analysis of the IPAV and 56601 strains

Individual protein abundances and the statistical significance of expression differences in orthologs identified from strains of IPAV and 56601 cultured in EMJH medium are listed in Supplementary information, Table S4. Among the selected 1627 pairs of orthologs, 149 proteins were upregulated in IPAV $(Z>1.96$, corresponding to $95 \%$ confidence), and 25 proteins were not detected in strain 56601; conversely, 187 proteins were upregulated $(Z<-1.96, P<0.05)$ in strain 56601, and 41 proteins were not observed in IPAV (Supplementary information, Table S4).

The upregulated proteins in 56601 were functionally enriched in the COG categories of translation and DNA replication/repair, whereas upregulated proteins in IPAV were enriched for the categories of energy production/ conversion, posttranslational processing and lipid metab- olism (chi-square test, $P<0.05$ ) (Figure 6). Among these genes, only 15 of these differentially expressed orthologs were found to have mutations in their upstream intergenic regions, while another 16 genes had mutations that occurred in their CDSs. Of these, the six in-frame deletions/insertions and five nonsynonymous SNVs might influence the stability of the protein. All of the remaining genes were free of mutations in their upstream intergenic or coding regions, and apparent variations in the expression level may be caused by mutations in transcriptional regulators or signal transduction systems controlling these genes.

In strain 56601, 17 proteins involved in translation were upregulated. These proteins included many ribosomal proteins, tRNA synthetases and the ribosomebinding factor, RbfA. Interestingly, we found that the BipA protein displayed a significant bias in expression between the two strains $\left(Z=7.74, P=9.77 \times 10^{-15}\right)$. A total of 171 peptides were mapped on BipA (LIF A1107) in strain IPAV, but only 17 peptides hit its ortholog in strain 56601, LA_1378. The BipA protein shares sequence similarity with EF-G and can interact with the $70 \mathrm{~S}$ ribosome, acting as a regulatory factor for normal protein synthesis. However, as was reported, overexpression of BipA will inevitably disrupt the normal func- 


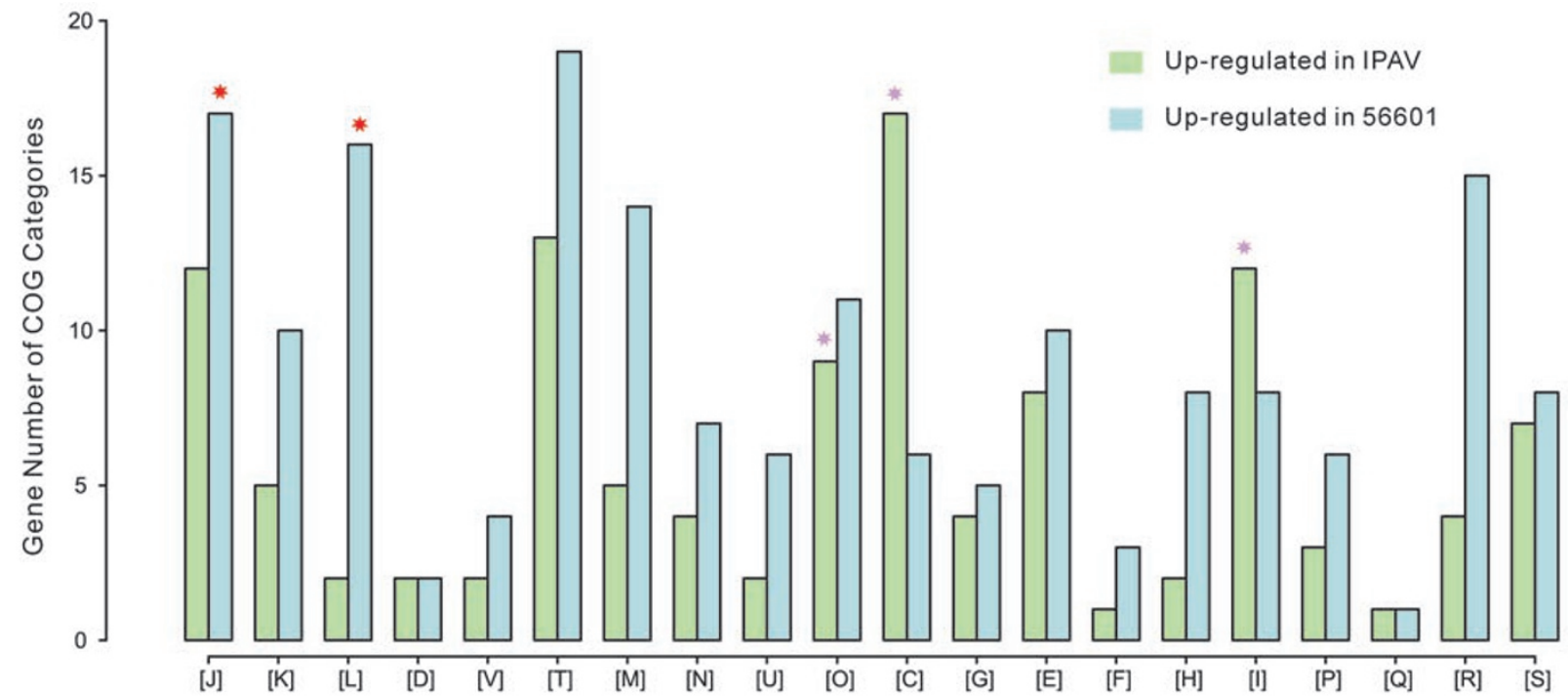

Figure 6 Functional classification of upregulated orthologs in IPAV and 56601. Green: upregulated genes in IPAV. Blue: upregulated genes in 56601. The upregulated genes include those orthologs that are only expressed in one of the strains. Proteins were clustered according to COG assignment. If a protein had more than one COG category, all were used. A purple/ red asterisk is situated in the functional category where the enrichment in IPAV/56601 was discovered by chi-square test.

tions of the ribosome and impair protein synthesis [24]. Because a dramatic difference in protein expression was observed, we postulated that the overexpression of BipA in IPAV might have a negative impact on protein translation and ribosomal biogenesis (shown by the comparative proteomic data). It is noteworthy that BipA in strain 56601 has a 56601-specific R66I nonsynonymous variation in the N-terminal P_loop_NTPase domain. Multiple sequence alignment of BipA from 50 different bacteria indicated that this mutated site is always a basic residue, such as Lys (25), Arg (4) or His (20). Therefore, this R66I mutation might be the cause of the lower level of BipA detected in the virulent strain 56601, which could ultimately lead to more active protein translation in this highly virulent strain.

Proteomic data indicated that purine/pyrimidine metabolism and DNA replication/repair in strain 56601 were much more active than in strain IPAV. Several enzymes involved in these pathways were upregulated in strain 56601, including DNA polymerase III, polymerase I, DNA helicase, DNA ligase, DNA gyrase and the mismatch repair protein MutL/MutS. In addition, the ribonucleotide-diphosphate reductase, $n r d A$ (LA_2360), which is responsible for the conversion of ribonucleotides into deoxyribonucleotides, was expressed approximately three times more in strain 56601 than its ortholog in IPAV. Interestingly, this rate-limiting DNA biosynthesis enzyme has a 56601-specific missense mutation causing an A703T substitution within a barrel domain. It was previously reported that, compared with other metabolic pathways, inactivation of purine/pyrimidine pathway genes in Escherichia coli and some other bacteria was the key factor for limiting their growth in human serum [25]. Therefore, these variations are likely, to a certain extent, to contribute to the avirulence property of strain IPAV.

Although not statistically enriched, another functional category exhibiting a notable bias in protein expression between the two strains was the cell envelope and outer membrane biogenesis category. In this class, proteins such as glycosyltransferase, $\mathrm{N}$-acetylneuraminic acid synthetase, lauroyl/myristoyl acyltransferase, UDPglucose 6-dehydrogenase, 3-deoxy-D-manno-octulosonic-acid transferase and CapA were upregulated in strain 56601. In addition, expression variation for many lipoproteins not classified in this category was also noticed (Table 3). For example, both the exclusively outer membrane lipoproteins, LipL48 $(Z=-15.33, P=0)$ and LipL36 $\left(P=3.63 \times 10^{-2}\right)$, maintained high expression levels in strain 56601 compared with those in IPAV. In contrast, significant increases in abundance were detected in IPAV compared with strain 56601 for LipL41 $(Z=$ $10.23, P=0)$, OmpL1 $(Z=17.61, P=0)$ and the peripheral membrane protein, $\operatorname{LipL} 45(Z=20.31, P=0)$.

Proteins involved in energy production, lipid metabolism and posttranslational processing were overrepresented in strain IPAV. They included a series of key enzymes in the citric-acid cycle including citrate synthase 
Table 3 Unequally expressed orthologs of OMPs and lipoproteins in IPAV and 56601

\begin{tabular}{|c|c|c|c|c|c|c|}
\hline \multicolumn{2}{|c|}{ L. interrogans strain IPAV } & \multicolumn{2}{|c|}{ L. interrogans strain 56601} & \multirow[b]{2}{*}{$Z$-score } & \multirow[b]{2}{*}{$P$-value } & \multirow[b]{2}{*}{ Gene product } \\
\hline Locus & Ave. abundance & Locus & Ave. abundance & & & \\
\hline$\overline{\text { LIF_A0412 }}$ & 2.72 & LA_0492 & 3.52 & -2.094 & $3.63 \mathrm{E}-02$ & LipL36 \\
\hline LIF_A1578 & 0.81 & LA_1957 & 1.54 & -2.229 & $2.58 \mathrm{E}-02$ & Putative lipoprotein \\
\hline LIF_A1809 & 0.48 & LA_2219 & 1.00 & -2.010 & $4.45 \mathrm{E}-02$ & Putative lipoprotein \\
\hline LIF_A2614 & 2.24 & LA_3262 & 4.32 & -2.589 & $9.63 \mathrm{E}-03$ & Putative lipoprotein \\
\hline LIF_A2871 & 0.17 & LA_3571 & 1.05 & -2.888 & $3.88 \mathrm{E}-03$ & Conserved hypothetical lipoprotein \\
\hline LIF_A2902 & 0.04 & LA_3611 & 0.24 & -2.578 & $9.93 \mathrm{E}-03$ & Putative lipoprotein \\
\hline LIF_B116 & 0 & LB_143 & 0.50 & - & - & LipL45-related protein \\
\hline LIF_A0125 & 1.73 & LA_0137 & 1.41 & 2.183 & $2.91 \mathrm{E}-02$ & Hypothetical lipoprotein \\
\hline LIF_A0506 & 39.84 & LA_0616 & 27.43 & 10.232 & 0 & LipL41 \\
\hline LIF_A1179 & 0.45 & LA_1468a & 0 & - & - & Putative lipoprotein \\
\hline LIF_A1561 & 3.73 & LA_1939 & 2.59 & 3.567 & $3.61 \mathrm{E}-04$ & Putative lipoprotein \\
\hline LIF_A1635 & 2.07 & LA_2023 & 1.04 & 2.958 & $3.10 \mathrm{E}-03$ & Putative lipoprotein \\
\hline LIF_A1636 & 5.09 & LA_2024 & 4.07 & 3.745 & $1.81 \mathrm{E}-04$ & Conserved hypothetical lipoprotein \\
\hline LIF_A1825 & 1.73 & LA_2240 & 0.25 & 2.464 & $1.37 \mathrm{E}-02$ & Hypothetical lipoprotein \\
\hline LIF_A1873 & 13.31 & LA_2295 & 3.94 & 20.305 & 0 & LipL45 \\
\hline LIF_A2387 & 1.65 & LA_2936 & 0.51 & 4.768 & $1.86 \mathrm{E}-06$ & LipL45-related lipoprotein \\
\hline
\end{tabular}

(LIF A0645), isocitrate dehydrogenase (LIF A3240), the succinyl-CoA synthetase alpha/beta subunits (LIF A0892/ LIF_A0893), fumarate hydratase (LIF_A0159) and malate dehydrogenase (LIF_A1738) as well as genes responsible for fatty acid/lipid metabolism, which generates acetyl-CoA for the initial step of the citric-acid cycle. However, there was no genetic mutation found in these genes with the exception of one coding for a longchain fatty acid CoA ligase. Therefore, we assume that mutations in trans-regulatory proteins might account for this phenomenon, as discussed in previous sections.

\section{Mutations affecting genes related to signal-induced regu- lation}

There is growing evidence that $\mathrm{Ser} / \mathrm{Thr}$ protein kinases (STPKs) also exist in the genomes of microbes in addition to their ubiquitous homologs in eukaryotes
[26]. Prokaryotes use STPKs and phosphatases not only to regulate many intracellular metabolic processes but also to control stress responses, cell density, cell segregation, carbon and nitrogen assimilation and virulence [27, 28]. In certain species, functionally related kinases and phosphatases are located in a single operon and are cotranscribed to maintain balanced expression and cooperative signal transduction activities for certain pathways [29]. Leptospira engages this module in one enzyme by fusing the kinase domain with the phosphatase domain. Although three copies of this kind of hybrid genes exist in the genome of strain 56601, only one (LA_1422, $1780 \mathrm{aa}$ ) is highly expressed (520 peptides identified), whereas the other two gene products (LA 1164 and LA 3113) are almost undetectable in our MS data. In fact, similar MS results can also be found in the work for Copenhageni $[20,30]$. Compared with the genes from 
strain 56601 and Copenhageni, a single nucleotide in the coding region is deleted in IPAV, leading to a frameshift and yielding two truncated CDSs (LIF_A1136, 930 aa and LIF_A1137, 851 aa). Moreover, we identified one peptide (K.TPVEQAER.I) matching the changed aminoacid residues in IPAV, LIF_A1137 (Figure 7A). Although a total of 160 peptides that contained a kinase domain mapped to LIF_A1137, no peptides were identified in LIF_A1136, which contains a PP2C phosphatase domain (Figure 7B). Given that the other two paralogs (LIF A0944 and LIF_A2517) are minimally expressed, it can be inferred that as the mutation occurred in strain IPAV the 3'-proximal phosphatase was no longer expressed, and its regulatory function was obviously impaired.

In prokaryotes, the two-component system (TCS) plays an essential role in signal transduction. When compared with four other pathogenic leptospires, the strain
IPAV histidine kinase LIF_A0838 has a point mutation at amino-acid residue 426, which results in replacement of Gly by Arg in a highly conserved ATP-binding site. Considering that autophosphorylation of the histidine kinase is dependent on this well-conserved ATP-binding domain [31] and that TCS not only regulates basic housekeeping functions but also governs toxins and other proteins important for pathogenesis [32], the mutation in LIF A0838 may partially account for the avirulence of IPAV.

\section{Mutations affecting genes related to stress response}

The $c s t$ genes, a group of cyclic AMP (cAMP)-dependent carbon-starvation response genes, are involved in response to nutrient-source deficiency [33, 34]. The results from previous studies indicated that the virulence factor CstA is involved in peptide transport, which could expand the range of substrates that a bacterium can uti-

A

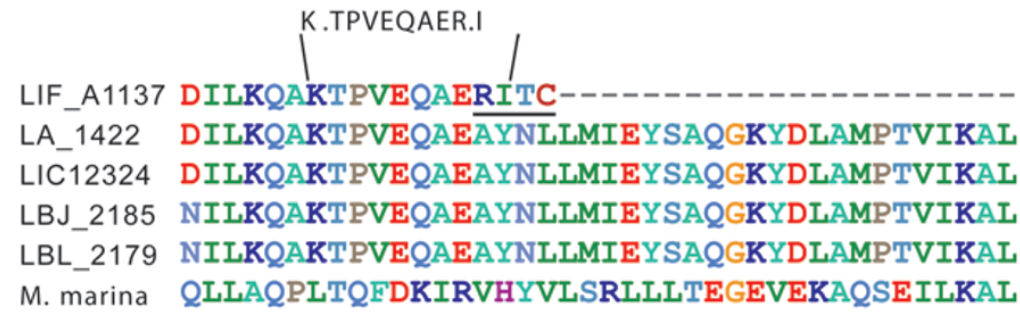

B

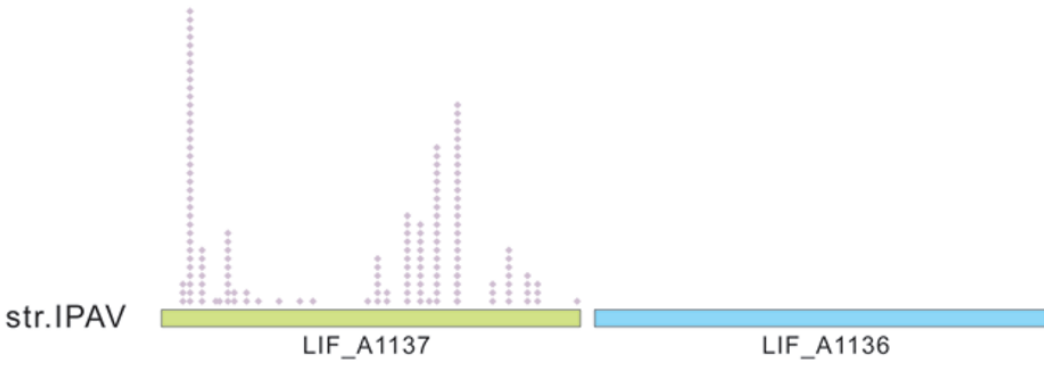

str. 56601

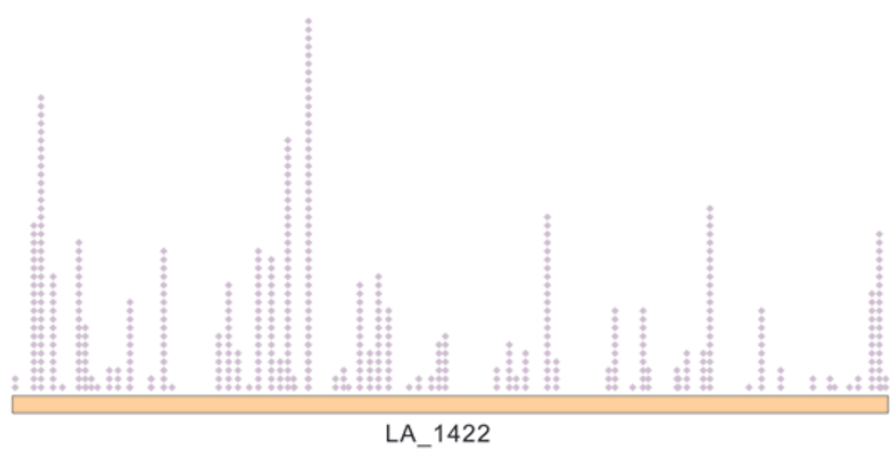

Figure 7 Comparison of the mutation-affected STPK in IPAV to its orthologs in other species. (A) Multiple sequence alignment of STPK sequences retrieved from NCBI (strain 56601, NP_711603.1; Fiocruz L1-130, YP_002258.1; JB197, YP_801432.1; L550, YP_798524.1; Microscilla marina, ZP_01687505.1). (B) MS-detected peptides mapping on the STPKs in IPAV and 56601. The bars with different colors represent the genes, and the purple dots above the genes represent the detected peptides at their corresponding sites. 
lize and can assist the cell in escaping carbon starvation $[35,36]$. Protein topology analysis on Leptospira CstA showed that it contained at least 15 transmembrane helices. This hydrophobicity profile agrees well with its function in peptide transport. However in IPAV, the cstA gene LIF A0252 has an in-frame deletion of 21 nucleotides between helixes 10 and 11, which leads to a 7-aa shorter product compared with the orthologous CstA in pathogenic strains 56601, Copenhageni and L. borgpetersenii. Notably, IPAV CstA was not identified by our MS data, whereas 16 peptides were found for the CstA in strain 56601. In light of this finding, we hypothesize that this critical mutation may influence the stability of the CstA protein. Additionally, the absence of the cstA gene in saprophytic $L$. biflexa suggests that it could be involved in the pathogenesis of $L$. interrogans.

Enzymes of the Rel/Spo family enable bacteria to survive during periods of nutrient limitation by producing an intracellular signaling molecule, (p)ppGpp, which triggers the so-called stringent response [37]. Previous studies demonstrated that the ability of bacteria to synthesize (p)ppGpp and mount a stringent response is an essential physiological adaptation required for stages of pathogenesis, such as initial growth of adherent bacteria [38], toxin production [39], motility [40] and expression of other virulence regulators [41]. Comparative analysis revealed that the IPAV spoT gene (LIF_A2491) has two missense mutations at the amino-acid residues, Val293 and Lys407, resulting in replacement by Ala and Glu, respectively. Apparently, the V293A mutation in the hydrolysis domain may have little impact on its function, as Val and Ala are both aliphatic amino acids. However, the K407E mutation occurred at a conserved site within the TGS motif and may impair the enzymatic function by decreasing the regulation efficiency of TGS toward both the N-terminal synthesis domain and the hydrolysis domains [42], eventually weakening the capacity for in vivo persistence and long-term survival of IPAV under starvation.

\section{Mutations affecting transmembrane channels}

The $\mathrm{H}^{+}$-translocating pyrophosphatase $\left(\mathrm{H}^{+}\right.$-PPase), which exists in a wide variety of organisms, is a membrane-bound proton pump that hydrolyzes inorganic pyrophosphate to generate a proton gradient across the plasma membrane $[43,44]$. In prokaryotes, the proton gradient is used mainly for intermediate energy storage, transportation of nutrients into the cell and flagellar rotation. According to the literature, $\mathrm{H}^{+}$-PPase always contains 14-16 transmembrane domains as well as several large cytoplasmic loops with important functional motifs [43]. Sequence alignment of $\mathrm{H}^{+}$-PPases from different organisms showed high similarity at the aminoacid level, especially in the carboxyl terminus. In IPAV,
A

LILI 634 -KAGGKGSDQQ̊KAAVVGDTVGDPFKDTSGP LILL 643 -KAGGKGSDQHKAAVVGDTVGDPFKDTSGP LICF 643 -KAGGKGSDQHKAAVVGDTVGDPFKDTSGP LBHL 643 -KTGGKGSDQHKAAVVGDTVGDPFKDTSGP LBHJ 643 -KTGGKGSDQHKAAVVGDTVGDPFKDTSGP LBPA 654 -TAGGKGSEKHKAAVVGDTVGDPFKDTSGP SC 687 -HHGGKGSEAHAATVIGDTVGDPFKDTAGP AT 710 KSLGPKGSEPHKAAVIGDTIGDPLKDTSGP

C LILI 175 RPGTKGGCFPVAPVDSOVDLRADIVKTLHO LILL 175 RPGTKGGYFPVAPVDSOVDLRADIVKTLHO LICF 175 RPGTKGGYFPVAPVDSOVDLRADIVKTLHO LBHL 175 RPGTKGGYFPVAPVDSQVDLRVAIVKTLHQ LBHJ 175 RPGTKGGYFPVAPVDSQVDLRIAIVKTLHQ LBPA 188 RPGTKGGYFPVAPIDSOVDLRAEFVKTLEA EC 173 RPAVKGGYFPVPPVDSAQDIRSEMCLVMEQ MT 179 KVRHKGGYFPVAPNDOYVDLRDKMLTNLIN
B $\begin{array}{lll}\text { LILI } & 80 & \text { QDLMFLFLAVMLTDIVLLDLYSTLGLPTST } \\ \text { LILL } & 70 & \text { QDLMFLFLAVMLTDIVLLDLYNTLGLPTST } \\ \text { LICF } & 80 & \text { QDLMFLFLAVMLTDIVLLDLYNTLGLPTST } \\ \text { LBHL } & 80 & \text { QDLMFLFLAVMLTDIVLLDLYNTLGLTST } \\ \text { LBHJ } & 80 & \text { QDLMFLFLAVMLTDIVLLDLYNTLGLPTST } \\ \text { BV } & 81 & \text { QELMCIFLAVMVTDVVLLDIFNSLGMPTST } \\ \text { FB } & 81 & \text { NEIMIIFMAVMITDILLLDFFNTLGMPTST } \\ \text { PG } & 81 & \text { SELMVVFLSVVITDVVLMDMFNSLGMPTST }\end{array}$

D LILI 196 SAGGIDSVVEII $\stackrel{*}{*}$ * LILL 196 SAGGIDSVVEILNLVDRGTEKTIIEALEEE LICF 196 SAGGIDSVVEILNLVDRGTEKTIIEALEEE LBHL 196 SAGGIDSVVEILNLVDRGTEKTIIEALEEE LBHJ 196 SAGGIDSVVEILNLVDRGTEKTIIEALEEE LBPA 196 SAGGIDSVVEILNLVYRGTEKTIIEALEEE TP 211 AAGGVQNIVDILNLVDRSSEKSIVEALEDE

BS 197 OTGGIEAVVEVLNGVDRGTEKTILDSLEIQ LILL: interrogans str. IPAV LILL: L.interrogans str. Lai LICF: $L$, interrogans str. Fiocruz L1-130 LBHL: L. borgpetersenii str. L550 LBHJ: L. borgpetersenii str. JB197 LBPA: L. biflexa str. Ames

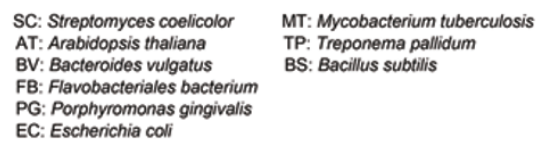
EC: Escherichia coli

Figure 8 Multiple sequence alignments of proton-PPase (A), phosphate sodium symporter (B), GInA (C) and FliG (D). Each site of substitution is indicated by an asterisk above the sequence. The numbers refer to the sites of amino-acid residues. The GenBank accession numbers of the used sequences are as follows: Streptomyces coelicolor (SC, NP_627745.1), Arabidopsis thaliana (AT, NP_173021.1), Bacteroides vulgatus (BV, YP_001299118.1), Flavobacteriales bacterium (FB, ZP_01105510.1), Porphyromonas gingivalis (PG, NP_906040.1), Escherichia coli (EC, NP_418306.1), Mycobacterium tuberculosis (MT, YP_001283561.1), Treponema pallidum (TP, NP_218840.1) and Bacillus subtilis (BS, NP_389504.1). 
the $\mathrm{H}^{+}$-PPase homolog, LIF_A1181, had a point mutation at the highly conserved residue 644 with a His substituted by a Gln in the C-terminus (Figure 8 ). This site is just three residues away from Ser641, which has been demonstrated to be essential for PPi hydrolysis by sitedirected mutagenesis studies in Streptomyces coelicolor [43]. This change in IPAV may imply that the function of the $\mathrm{H}^{+}$-PPase as an energy source for secondary active transport systems and a scavenger of cytoplasmic PPi may be impaired.

In L. interrogans, a sodium-dependent phosphate transporter gene was identified by similarity search. Homologous proteins found in Saccharomyces cerevisiae and Neurospora crassa were designated high-affinity phosphate sodium symporters and were induced under phosphate-limiting conditions to assist the organisms in survival during phosphate starvation [45, 46]. In IPAV, this phosphate sodium symporter (LIF_A2090) has an N101S mutation located in a highly conserved transmembrane helix in the PHO4 domain (Figure 8). Significantly, a sharp decrease in protein abundance of this symporter was observed in IPAV when compared to strain 56601. A total of 216 peptides mapped to this protein in 56601, but only 52 peptides were detected for LIF A2090 in IPAV. No mutation occurred in its predicted promoter region, and whether the alteration in the CDS caused the difference in expression remains to be determined. Nonetheless, this high-affinity symporter does not exist in the non-pathogenic $L$. biflexa, indicating its essential role in phosphorus uptake when pathogenic Leptospira encounter a phosphate-limiting condition in vivo.

Mutations affecting genes related to metabolism, cell division and motility

Glutamine, a source of nitrogen in the biosynthesis of some amino acids and many other metabolites, is produced by glutamine synthetase encoded by $g \ln A$, which catalyzes the ATP-dependent condensation reaction of ammonium and glutamate. Comparison of multiple sequences between Leptospira and other bacteria revealed that the glutamine synthetase of IPAV (LIF_A1058) had a point mutation at amino-acid residue 182 that led to replacement of a highly conserved Tyr with a Cys (Figure 8 ). According to a study of the glutamine synthetases of $S$. typhimurium and $M$. tuberculosis, this Tyr is involved in forming a pocket and directly functions as one of the ammonium binding sites [47, 48]. Therefore, we might infer that the ability to synthesize glutamine in IPAV may be impaired due to a decrease in the ammonium substratebinding efficiency. In addition, the expression of $g \ln A$ in strain 56601 and IPAV exhibited a distinctive feature revealed by our proteomic study: a total of 3341 pep- tides mapped to the $56601 \mathrm{G} \ln \mathrm{A}$, but only 702 peptides mapped to its ortholog in IPAV. This phenomenon is consistent with a previous report, which showed that the expression levels of $g \ln A$ are significantly greater in the pathogenic serovars [49].

Bacteria employ different types of genes to complete cell division for their continuous survival. In L. interrogans, we identified four peptidoglycan (PG) hydrolases containing peptidase M23 family domains, as well as one or two N-terminal LysM motifs, which have been demonstrated to have the ability to bind various types of peptidoglycans [50]. There is evidence that in Staphylococcus aureus, a PG hydrolase acts as a virulence factor that digests peptidoglycans during the cell division separation and assists in the dissemination of daughter cells in the host [51]. It was also reported that a PG hydrolase in Chlamydia pneumoniae was upregulated during longterm chronic infection [52]. In IPAV, the smallest PG hydrolase (LIF_A0265) has a G146V mutation located in the conserved peptidase domain. None of the four gene products was identified by MS in strains 56601 or IPAV cultured in the laboratory, and whether the PG hydrolase is expressed in L. interrogans and the LIF_A0265 mutation affects its hydrolase activity are worthwhile subjects for further analysis.

The turning of bacterial flagella is controlled by a rotary motor switch, a complex apparatus that is composed of three proteins: FliG, FliM and FliN [53]. Previous studies have demonstrated that the middle domain of FliG is important for flagellar assembly, and that mutations in or around a well-conserved hydrophobic patch from Ile198 to Ile231 directly weaken binding to FliM [54]. In IPAV, FliG (LIF_A0023) has two neighboring mutations, N208D and $\mathrm{D} 211 \mathrm{G}$, located in the conserved hydrophobic patch in the middle third of this protein (Figure 8). Active motility of strain 56601 was observed under a dark-field microscope as compared to avirulent IPAV and L. biflexa in vitro. Interestingly, although a conserved Asp residue dominated among the proteins from different genera, the corresponding second mutation site in L. biflexa is a Tyr.

\section{Mutations affecting genes related to degradation}

ADP-ribose is an intermediate that is produced during the metabolism of $\mathrm{NAD}^{+}$, mono- or poly-ADP-ribosylated proteins and cyclic ADP-ribose. A high concentration of ADP-ribose in the cell causes nonenzymatic ADPribosylation, which inactivates various proteins and interferes with metabolic regulation via enzymatic ADPribosylation $[55,56]$. The ADP-ribose pyrophosphatases (ADPRases), which constitute a subfamily of the Nudix hydrolases, hydrolyze ADP-ribose to AMP and ribose- 
5-phosphate. In IPAV, the ADPRase (LIF_A0876) has a point mutation in the conserved amino-acid residue 148 that leads to a substitution of acidic Asp by nonpolar Gly in the C-terminal domain. According to the structural study of the ADPRase of Mycobacterium tuberculosis (its corresponding amino acid is also an acidic residue), this site forms a conserved loop that contacts the substrate, triggers a conformation change of the enzyme and finally accomplishes catalysis [57]. Therefore, the alteration in IPAV ADPRase is likely to be deleterious, pending experimental confirmation.

ClpA, a Hsp100/Clp chaperone and an integral component of the ATP-dependent ClpAP protease, participates in regulatory protein degradation and the dissolution of protein aggregates [58]. In Leptospira, ClpA contains an $\mathrm{N}$-terminal domain consisting of two short-tandem Clp $\mathrm{N}$ motifs and two $\mathrm{AAA}^{+}$modules (ATPase) head-to-tail from the middle to the $\mathrm{C}$-terminal end. In the literature, the two ATPase domains of E. coli ClpA play different roles: the first ATPase domain (D1 in ClpA) mainly contributes to complex assembly/stability, while the second one (D2 in ClpA) possesses higher ATPase activity and contacts ClpP [59]. The IPAV clpA (LIF_A0333) has a missense A303T mutation in the D1 domain, where a functionally important loop related to conformational variability resides [59]. If Leptospira $\operatorname{clp} A$ has a function similar to that of its ortholog in E. coli, one may presume that the mutation in IPAV might affect ClpAP structure and consequently impair its activity.

\section{1-specific mutations}

Among all of the mutated genes found between IPAV and strain 56601, 13 genes with biological functions (not including previously described genes located in the large sequence deletions) contain 56601-specific mutations when their corresponding sites are compared to Copenhageni (Table 2), including trmA, cyoB, pts $N$, bipA, $n r d A$, fliF, thiM and acrR. Because Copenhageni is also a pathogenic strain, these 56601-specific mutations may not contribute directly to its virulence. However, because many of the mutated genes do not have a paralog in the genome of 56601, mutations that occur in conserved sites may influence the function of their gene products. We indeed found altered expression for some of these genes in the proteomic analysis between IPAV and strain 56601.

\section{Discussion}

The virulence-attenuated $L$. interrogans strain IPAV, assigned to serovar Lai by repeated serological tests, was derived from a virulent strain isolated from a Chinese patient whose clinical record has been unrecoverable thus far. Its characteristic avirulence was described in leptospirosis animal models followed by histological confirmation. In contrast to strain 56601, strain IPAV could not cause conspicuous pathological changes in the liver, kidney and lung tissues, and no clinical manifestations could be detected in any animal inoculated with a lethal dose intraperitoneally. Thus, a comparative study between stain IPAV and, the previously sequenced, highly virulent Lai-type strain 56601 was performed with the aim of elucidating the genetic basis for virulence.

Because we determined the entire genome sequence of strain IPAV using a high-density pyrosequencing method [11], all of the single-nucleotide indels residing in homopolymers were reevaluated against the sequence of the 56601 genome. Ambiguous bases were resequenced by conventional Sanger method using the corresponding PCR products as the templates. Meanwhile, some sequencing errors that existed in the published genome of strain 56601 were resolved in this study, and the genome was completely reannotated.

Comparative genomic analysis indicated that the two strains shared highly similar genome structures and gene orders; 33 insertions, 53 deletions, 301 SNVs and 3 LSPs were observed when IPAV was compared with strain 56601. It was particularly interesting to identify a $14-\mathrm{kb}$ DNA fragment, containing nine genes, in IPAV that was absent in strain 56601 and thus obviously not involved in virulence determination. This fragment also existed in the $L$. interrogans serovar Copenhageni but was absent in the parasitic $L$. borgpetersenii and the saprophytic $L$. biflexa. Further molecular epidemiological investigation is needed to trace its phylogeny in different species of $L$. interrogans. This result implied that the ancestral strain of IPAV was unlikely to be directly derived from 56601 . Other supporting evidence includes the partial deletion of a Type I R/M gene cluster uniquely found in 56601 among all of the Leptospira species that have been sequenced and the presence of five ISs (one IS1500 and two ISlin1 in IPAV; two ISlin1 in strain 56601) that are different between the two strains.

In this study, proteogenomics - a comprehensive strategy combining whole genome sequencing with proteomic studies [19, 60-62] - was employed for genome annotation in both strains. Quantitative proteomic studies for the two strains indicated that approximately half of the predicted proteins were validated $(55.8 \%$ for strain IPAV and 59.8\% for strain 56601), slightly more than that in recent studies for serovar Pomona [63] and Copenhageni [30]. Moreover, by searching the MS spectra against the six-frame translations of the genome sequence, 13 novel genes were detected. Many of these genes overlapped with their flanking neighbors, and 
as a result, were missed by the gene-calling programs. Hypothetical proteins are either real products that are unique to each organism or false hits that are predicted by the greedy gene-calling algorithms. From MS-based proteomic data, 651 hypothetical proteins $(46.3 \%$ of all hypothetical proteins) were detected with at least two unique peptides mapped in the L. interrogans strains. These results point to the importance of applying MS data in the validation of gene annotations, which is often lacking from genome studies alone. The lower confirmation rate of hypothetical proteins compared with those in COG categories could be explained by one or more of the following reasons: (1) hypothetical proteins are often expressed at lower concentrations and thus are less likely to be detected by MS, (2) hypothetical proteins have "uncommon" functions that might be induced under specialized conditions different from our studies, and (3) the gene-calling programs are biased in their statistics models toward predicting COG genes, which needs to be addressed by statistical analysis.

A comparative proteogenomic strategy was employed to further analyze the probable genetic factors that may contribute to the virulence-attenuation of strain IPAV. First, the probable effect of transposable elements related to the avirulence of IPAV was excluded because all of the indels caused by ISs that differed between the two strains were focused in intergenic regions.

Although we observed a significant proportion (more than $20 \%$ of all functional orthologs) of changes in the proteomic profiles of the two strains under laboratory growth conditions, only a few of these changes could be directly accounted for by nonsynonymous mutations identified in the corresponding genes. Therefore, genetic variation in regulatory proteins is likely the major cause of the global change in expression profiles, which in turn may eventually affect the virulence attenuation of IPAV in combination with other mechanisms, such as direct mutations in some virulence-related genes.

Possible genetic variations affecting the vitality of the cells may play a significant role in virulence determination. According to the MS data, both protein translation and DNA replication/repair were more active in strain 56601. Considering the 56601-specific mutations and distinct abundance of orthologous proteins observed for BipA, regulating the protein synthesis, and $\mathrm{NrdA}$, the rate-limiting enzyme for DNA biosynthesis, we assumed that these variations may alter the primary physiology of the two strains leading to the higher activity and vitality of strain 56601 and making it favorable for pathogenesis during infection. In addition, owing to the mutation at the conserved substrate binding site and the sharp decrease of protein abundance of glutamine synthetase GlnA, nitrogen metabolism or glutamine biosynthesis in IPAV must be largely impaired compared with 56601. Meanwhile, mutations in two starvation response genes, cst $A$ and spo $T$, may also directly decrease resistance ability to the stress imposed by an unfavorable host environment during infection.

Several genes involved in signal transduction and transcription regulation were found to have genetic variations in IPAV or 56601. These included a signal transduction-related STKP, a TCS gene, an anti-sigma factor antagonist and an AcrR trans-acting factor. According to this study, a nucleotide deletion in the STKP may severely influence the cellular process in IPAV, due to its truncation and the loss of the phosphatase function. Three other trans-regulators were affected with nonsynonymous SNV in coding regions. As revealed by the previous work of Yin-yang MDLC-MS/MS, the anti-sigma factor antagonist (LA_3096) can be phosphorylated in vivo [17], indicating an essential role in transcription regulation. These signal transduction pathways regulate a wide variety of intracellular processes in bacteria and sometimes are essential for coordinating responses to environmental changes. Given the fundamental importance of these genes, it is likely that the genetic mutations in these genes seriously impact the ability for strain IPAV to adapt inside host bodies (e.g., evading immune response or responding to environmental changes in host), thus resulting in virulence attenuation in strain IPAV.

Finally, we observed that some cell-surface proteins were either mutated or had alterations in the protein profiles. Two transmembrane channels, $\mathrm{H}^{+}$-PPase and phosphate sodium symporter, both contained IPAV-specific SNV mutations, but the latter had a dramatic decrease in its protein abundance in IPAV. We also observed high levels of expression of virulence-related proteins belonging to the TolC family and some $\mathrm{ABC}$ transporters, which interact in combination to facilitate the efflux of cytoplasmic components [64]. In addition, a TonB-like protein and a TonB-dependent outer membrane receptor were upregulated in strain 56601, indicating the intensified ability to uptake certain iron sources [65] from the environment to help its growth. LigB was reported to bind fibronectin during infection [66, 67]. However, mutagenesis of the gene by allelic exchange did not affect the virulence of the species [68]. We detected its expression under the laboratory culture conditions, but only in the pathogenic strain of 56601 (LA_3778). Whether it contributes to the virulence requires in-depth investigation. Notably, some outer-membrane proteins and lipoproteins were expressed unequally in the two strains, such as OmpL1, LipL45, LipL48, LipL41 and LipL36. These membrane constituents, the predominant leptospi- 
ral surface-exposed proteins, play pivotal roles in hostcell interaction, and some of them have been verified as being essential for in vivo infection of pathogenic leptospires [69]. These differences in expression ought to account for the avirulence of IPAV to a large extent and are likely caused by alterations in signal transduction and/or transcription regulation, as mentioned above.

\section{Materials and Methods}

\section{Bacteria}

L. interrogans serovar Lai strain IPAV was a gift from Pro. I. Saint-Girons and M. Picardeau. L interrogans serovar Lai strain 56601 was obtained by the Institute for Infectious Disease Control and Prevention, Beijing, China, and was maintained by serial passages in guinea pigs for the preservation of virulence. Both strains were grown in liquid Ellinghausen-McCullough-Johnson-Harris (EMJH) medium at $28{ }^{\circ} \mathrm{C}$ with shaking under aerobic conditions and simultaneously collected at mid-log phase at the density of $3 \times$ $10^{8}$ bacteria per $\mathrm{ml}\left(\mathrm{OD}_{600} \approx 0.075\right)$ for further genome sequencing and MS analysis.

\section{Histopathology studies}

The animal study was approved by the animal research committee of the Shanghai Jiao Tong University School of Medicine. For each strain, 25 guinea pigs of either sex were assigned to five groups. All animals were injected intraperitoneally with $1 \mathrm{ml}$ of 56601 or IPAV culture $\left(3 \times 10^{8}\right)$ and then euthanized after defined time periods. Tissues from lung, liver and kidney were collected from guinea pigs for histology studies. After fixing in neutralbuffered $4 \%$ formaldehyde, the processed tissues were stained by $\mathrm{H} \& \mathrm{E}$ according to routine histological procedures.

\section{Genome sequencing}

For genome sequencing, $5 \mu \mathrm{g}$ of genomic DNA of strain IPAV was extracted from cells. The large-scale IPAV genome sequencing was performed on a GS 20 system [11]. A total of 1.8 million reads were generated by 454 GS systems in three runs, and 872719 high-quality reads were obtained using the system filter criteria. The high-quality reads (813 924, 93\%) were assembled with the 454 assembly tools, which had an average depth of 17fold coverage of the genome and yielded 996 contigs. Among these, 448 large contigs, which ranged from 610 bp to 72277 bp with an average length of $10114 \mathrm{bp}$, represented $98 \%$ of the draft sequence. In the finishing process, the order of the selected large contigs was determined using the Blast program with the original published genome sequence of strain 56601. Physical gaps were filled through sequencing PCR products that spanned these regions using ABI 3730 xl DNA sequencers. Sequence assembly was accomplished by the Phred/Phrap/Consed software package [70, 71]. A gap that exceeded $6 \mathrm{~kb}$ and five gaps that contained multiple copies of tandem repeats were directly filled using a shotgun strategy. To ensure final accuracy, errors in the homopolymer sites that arose from the pyrosequencing method were resolved via comparison with the corresponding sites on 56601, and the ambiguous bases were resequenced using the ABI 3730 xl DNA sequencer. This traditional sequencing method was also used to find and re- vise sequence errors in strain 56601 when the chromosome-bychromosome alignment generated inconsistent matches between the two strains.

\section{Sequence annotation and analysis}

Open reading frames (ORFs) were identified by GLIMMER [72] and GeneMark [73]. The exact predicted CDSs and all six-frame CDSs were translated into proteins and used as the database for mass spectra searching, respectively. After incorporating the two search results, functional annotation was done by comparison with the in-house NCBI NR protein database using BLASTP, followed by a manual check. COG functional classification for each gene was assigned using RPS-BLAST against the CDD database [74]. Transfer RNA genes were identified with tRNAscan-SE [75]. Considering that the original sequence of 56601 was revised in this work, we reannotated this genome in parallel with IPAV (reannotation for strain 56601 has been done before [6, 17], both of which were based on the old version of genome sequence). Comparison of the chromosome sequences was performed using ACT [76]. A phylogenic tree based on the ISlin1 sequences was constructed using NJ methods of the MEGA package [77], and the reliability of each branch was tested by 1000 bootstrap replications.

\section{Gel electrophoresis separation and in-gel proteolysis}

The protein samples from strains 56601 and IPAV were prepared as follows. The harvested cells were suspended in lysis buffer (2\% SDS, $50 \mathrm{mM}$ Tris-HCL, $2 \mathrm{mM}$ PMSF, $2 \mathrm{mM}$ sodium fluoride, $2 \mathrm{mM}$ sodium orthovanadate) and sonicated. After centrifuging, the concentration of protein extract was determined by the bicinchoninic acid assay. Samples of $200 \mu \mathrm{g}$ were subjected to SDS-PAGE on $12.5 \%$ gels. The gels were entirely stained with Coomassie blue and excised into 22 sections, which were subjected to in-gel digestion with trypsin as described previously [78].

\section{$L C-M S / M S$}

Liquid chromatography was performed on a Surveyor liquid chromatography system coupled to a LTQ linear ion trap mass spectrometer (Thermo Fisher). The HPLC solvents used were $0.1 \%$ formic acid (v/v) aqueous (A) and $0.1 \%$ formic acid (v/v) acetonitrile (B). The proteolytic peptide mixtures were eluted using a gradient of $2 \%-40 \% \mathrm{~B}$ for $130 \mathrm{~min}$. The temperature of the heated capillary was set at $170{ }^{\circ} \mathrm{C}$. A voltage of $3.0 \mathrm{kV}$ was applied to the ESI needle. The normalized collision energy was $35.0 \%$. The mass spectrometer was set as one full MS scan (400-1800 m/z) followed by MS/MS of the 10 most intense ions. A dynamic exclusion of $120 \mathrm{~s}$ was applied.

\section{MS/MS data analysis}

After two independent biological replicates were performed, the acquired MS/MS spectra were searched against the databases consisting of forward and reverse sequences of all six-frame CDSs and the final confirmed CDSs using the TurboSEQUEST program in the BioWorks 3.2 software package. The following search criteria were employed: full tryptic specificity was required and two missed cleavages were allowed, carbamidomethylation was set as a fixed modification and oxidation (M) was set as a modification variable, and precursor and fragment ion mass tolerances were 3.0 Da and 1.0 Da (default), respectively. A cutoff of $1.0 \%$ falsepositive ratio (FPR) [79] and at least 0.1 DeltaCn were set to filter 
the identified peptides (for raw MS results refer to Supplementary information, Dataset S1-S4). To evaluate the concordance between the two replicates in IPAV and 56601, Pearson correlation tests were performed by calculating identified peptides of the whole proteins in the two parallel datasets. The Pearson correlations are 0.87754 and 0.8685888 for IPAV and strain 56601, respectively.

\section{Protein quantification and data comparisons between the two strains}

We chose the proteins assigned by at least two unique peptides to perform the quantification. Protein abundance was calculated mainly based on the simplified APEX-index [80] with slight modification. We utilized the in silico predicted proteotypic peptides to substitute the previously used theoretical tryptic peptides. The protein abundance was defined as:

$$
\text { Abundance }=\mathrm{PEP}_{\mathrm{I}} \times\left(\frac{\mathrm{PT}_{\mathrm{I}} \times \mathrm{PEP}_{\mathrm{MIX}}}{\mathrm{PT}_{\mathrm{MIX}}}\right)^{-1},
$$

where $\mathrm{PEP}_{\mathrm{I}}$ and $\mathrm{PEP}_{\mathrm{MIX}}$ are the number of detected peptides of an individual protein and the sample of protein mixtures, respectively. $\mathrm{PT}_{\mathrm{I}}$ and $\mathrm{PT}_{\mathrm{MIX}}$ represent the number of in silico predicted proteotypic peptides of an individual protein and the sample of protein mixtures, respectively. The proteotypic peptides were predicted using PeptideSieve software [81]. The parameters were set as follows: experimental design (PAGE_ESI), peptide length (5-50), peptide mass (400-6000), allowed mis-cleavage $(<2)$ and threshold score (60).

To ensure the high confidence of the comparisons of the proteomic data, we chose orthologous proteins that both had more than two unique peptides, and at least one of the two orthologs had more than 10 total peptides to perform the comparison. A statistical formulation, assuming protein expression profiling that is well approximated by a normal distribution described previously [82, 83], was introduced to measure which pairs of orthologs were significantly differentially expressed in the two strains:

$$
Z=\frac{\mathrm{P}_{\mathrm{i} \text { IPAV }}-\mathrm{P}_{\mathrm{iLai}}}{\sqrt{\mathrm{P}_{\mathrm{i} 0}\left(1-\mathrm{P}_{\mathrm{i} 0}\right) / \mathrm{PEP}_{\mathrm{MIX} \text { IPAV }}+\mathrm{P}_{\mathrm{i} 0}\left(1-\mathrm{P}_{\mathrm{i} 0}\right) / \mathrm{PEP}_{\mathrm{MIX} \mathrm{Lai}}}}
$$

Briefly, $\mathrm{P}_{\mathrm{i}}=\mathrm{PEP}_{\mathrm{I}} / \mathrm{PEP}_{\mathrm{MIX}}$, so the numerator is the difference in proportions of orthologous proteins in IPAV and Lai 56601 (abbreviated as "Lai" in the equation), and $\mathrm{P}_{\mathrm{i} 0}=\left(\mathrm{PEP}_{\mathrm{I} \text { IPAV }}+\mathrm{PEP}_{\mathrm{I} \mathrm{Lai}}\right) /$ $\left(\mathrm{PEP}_{\mathrm{MIX} \text { IPAV }}+\mathrm{PEP}_{\mathrm{MIX} \mathrm{Lai}}\right)$, so the denominator is the standard error of the difference if the null hypothesis is true. Using the normally distributed $Z$ statistic, different expression of orthologous proteins can be decided by $Z>1.96$ or $<-1.96(P<0.05)$. Additionally, proteins that had fewer than two unique peptides but were observed in the other strain with more than two unique peptides and 10 total peptides were defined as non-expressed proteins in one strain but expressed in the other.

Functional distribution of the strain-specific upregulated proteins was classified according to COG functional categories. The enrichment of certain categories was evaluated using the chisquare test, and significant classes with $P<0.05$ were selected as overrepresented.

\section{Competing interests}

The authors have declared that no competing interests exist.

\section{Acknowledgments}

We thank Professor M Picardeau for the gift of L. interrogans strain IPAV and Bao-Yu Hu and Yang Yang for the support in performing bacteria culture. We also thank Dr Junwei Yang for suggestions in revising the manuscript. We thank the genome sequencing team at Chinese Human Genome Center at Shanghai for their assistance. This work was supported in part by grants from the National Natural Science Foundation of China (30770111, 30900051 and 30970125), the National Key Program for Infectious Diseases of China (2008ZX10004 and 2009ZX10004), the Program of Shanghai Subject Chief Scientist (09XD1402700) and the Program of Shanghai Research and Development (10JC1408200).

\section{References}

1 Paster BJ, Dewhirst FE, Weisburg WG, et al. Phylogenetic analysis of the spirochetes. J Bacteriol 1991; 173:6101-6109.

2 Haake DA. Spirochaetal lipoproteins and pathogenesis. $\mathrm{Mi}$ crobiology 2000; 146 (Pt 7):1491-1504.

3 Bharti AR, Nally JE, Ricaldi JN, et al. Leptospirosis: a zoonotic disease of global importance. Lancet Infect Dis 2003; 3:757-771.

4 Ko AI, Goarant C, Picardeau M. Leptospira: the dawn of the molecular genetics era for an emerging zoonotic pathogen. Nat Rev Microbiol 2009; 7:736-747.

5 Faine S, Adler B, Bolin C, Perolat P. Leptospira and Leptospirosis. Melbourne: Medisci, 1999.

6 Bulach DM, Zuerner RL, Wilson P, et al. Genome reduction in Leptospira borgpetersenii reflects limited transmission potential. Proc Natl Acad Sci USA 2006; 103:14560-14565.

7 Ren SX, Fu G, Jiang XG, et al. Unique physiological and pathogenic features of Leptospira interrogans revealed by whole-genome sequencing. Nature 2003; 422:888-893.

8 Murray GL, Srikram A, Henry R, et al. Leptospira interrogans requires heme oxygenase for disease pathogenesis. Microbes Infect 2009; 11:311-314.

9 Murray GL, Morel V, Cerqueira GM, et al. Genome-wide transposon mutagenesis in pathogenic Leptospira species. Infect Immun 2009; 77:810-816.

10 Zapata S, Trueba G, Bulach DM, et al. Characterization of a lipopolysaccharide mutant of Leptospira derived by growth in the presence of an anti-lipopolysaccharide monoclonal antibody. FEMS Microbiol Lett 2010; 309:144-150.

11 Margulies M, Egholm M, Altman WE, et al. Genome sequencing in microfabricated high-density picolitre reactors. Nature 2005; 437:376-380.

12 Ristow P, Bourhy P, da Cruz McBride FW, et al. The OmpAlike protein Loa22 is essential for leptospiral virulence. PLoS Pathog 2007; 3:e97.

13 Yang HL, Jiang XC, Zhang XY, et al. Thrombocytopenia in the experimental leptospirosis of guinea pig is not related to disseminated intravascular coagulation. BMC Infect Dis 2006; 6:19.

14 Yang J, Zhang Y, Xu J, et al. Serum activity of plateletactivating factor acetylhydrolase is a potential clinical marker for leptospirosis pulmonary hemorrhage. PLOS ONE 2009; 4:e4181.

15 Tatusov RL, Koonin EV, Lipman DJ. A genomic perspective 
on protein families. Science 1997; 278:631-637.

16 Bourhy P, Salaun L, Lajus A, et al. A genomic island of the pathogen Leptospira interrogans serovar Lai can excise from its chromosome. Infect Immun 2007; 75:677-683.

17 Cao XJ, Dai J, Xu H, et al. High-coverage proteome analysis reveals the first insight of protein modification systems in the pathogenic spirochete Leptospira interrogans. Cell Res 2010; 20:197-210.

18 Jaffe JD, Berg HC, Church GM. Proteogenomic mapping as a complementary method to perform genome annotation. Proteomics 2004; 4:59-77.

19 Jaffe JD, Stange-Thomann N, Smith C, et al. The complete genome and proteome of Mycoplasma mobile. Genome Res 2004; 14:1447-1461.

20 Malmstrom J, Beck M, Schmidt A, et al. Proteome-wide cellular protein concentrations of the human pathogen Leptospira interrogans. Nature 2009; 460:762-765.

21 Nascimento AL, Ko AI, Martins EA, et al. Comparative genomics of two Leptospira interrogans serovars reveals novel insights into physiology and pathogenesis. J Bacteriol 2004; 186:2164-2172.

22 Piekarowicz A, Klyz A, Kwiatek A, Stein DC. Analysis of type I restriction modification systems in the Neisseriaceae: genetic organization and properties of the gene products. $\mathrm{Mol}$ Microbiol 2001; 41:1199-1210.

23 Murray GL, Srikram A, Henry R, et al. Mutations affecting Leptospira interrogans lipopolysaccharide attenuate virulence. Mol Microbiol 2010; 78:701-709.

24 Owens RM, Pritchard G, Skipp P, et al. A dedicated translation factor controls the synthesis of the global regulator Fis. Embo J 2004; 23:3375-3385.

25 Samant S, Lee H, Ghassemi M, et al. Nucleotide biosynthesis is critical for growth of bacteria in human blood. PLoS Pathog 2008; 4:e37.

26 Kannan N, Taylor SS, Zhai Y, Venter JC, Manning G. Structural and functional diversity of the microbial kinome. PLoS Biol 2007; 5:e17.

27 Shi L. Manganese-dependent protein O-phosphatases in prokaryotes and their biological functions. Front Biosci 2004; 9:1382-1397.

28 Wehenkel A, Bellinzoni M, Grana M, et al. Mycobacterial Ser/Thr protein kinases and phosphatases: physiological roles and therapeutic potential. Biochim Biophys Acta 2008; 1784:193-202.

29 Hussain H, Branny P, Allan E. A eukaryotic-type serine/threonine protein kinase is required for biofilm formation, genetic competence, and acid resistance in Streptococcus mutans. J Bacteriol 2006; 188:1628-1632.

30 Eshghi A, Cullen PA, Cowen L, Zuerner RL, Cameron CE. Global proteome analysis of Leptospira interrogans. J Proteome Res 2009; 8:4564-4578.

31 Wolanin PM, Thomason PA, Stock JB. Histidine protein kinases: key signal transducers outside the animal kingdom. Genome Biol 2002; 3:REVIEWS3013.

32 Lamarche MG, Wanner BL, Crepin S, Harel J. The phosphate regulon and bacterial virulence: a regulatory network connecting phosphate homeostasis and pathogenesis. FEMS Microbiol Rev 2008; 32:461-473.

33 Matin A. The molecular basis of carbon-starvation-induced general resistance in Escherichia coli. Mol Microbiol 1991; 5:3-10.

34 Matin A. Role of alternate sigma factors in starvation protein synthesis--novel mechanisms of catabolite repression. Res Microbiol 1996; 147:494-505.

35 Schultz JE, Matin A. Molecular and functional characterization of a carbon starvation gene of Escherichia coli. J Mol Biol 1991; 218:129-140.

36 Tenor JL, McCormick BA, Ausubel FM, Aballay A. Caenorhabditis elegans-based screen identifies Salmonella virulence factors required for conserved host-pathogen interactions. Curr Biol 2004; 14:1018-1024.

37 Hogg T, Mechold U, Malke H, Cashel M, Hilgenfeld R. Conformational antagonism between opposing active sites in a bifunctional RelA/SpoT homolog modulates (p)ppGpp metabolism during the stringent response. Cell 2004; 117:57-68.

38 Taylor CM, Beresford M, Epton HA, et al. Listeria monocytogenes relA and $h p t$ mutants are impaired in surface-attached growth and virulence. J Bacteriol 2002; 184:621-628.

39 Haralalka S, Nandi S, Bhadra RK. Mutation in the relA gene of Vibrio cholerae affects in vitro and in vivo expression of virulence factors. $J$ Bacteriol 2003; 185:4672-4682.

40 Erickson DL, Lines JL, Pesci EC, Venturi V, Storey DG. Pseudomonas aeruginosa relA contributes to virulence in Drosophila melanogaster. Infect Immun 2004; 72:5638-5645.

41 Pizarro-Cerda J, Tedin K. The bacterial signal molecule, ppGpp, regulates Salmonella virulence gene expression. Mol Microbiol 2004; 52:1827-1844.

42 Potrykus K, Cashel M. (p)ppGpp: still magical? Annu Rev Microbiol 2008; 62:35-51.

43 Mimura H, Nakanishi Y, Hirono M, Maeshima M. Membrane topology of the H+-pyrophosphatase of Streptomyces coelicolor determined by cysteine-scanning mutagenesis. $J$ Biol Chem 2004; 279:35106-35112.

44 Lin HH, Pan YJ, Hsu SH, et al. Deletion mutation analysis on C-terminal domain of plant vacuolar $\mathrm{H}(+)$-pyrophosphatase. Arch Biochem Biophys 2005; 442:206-213.

45 Persson BL, Petersson J, Fristedt U, et al. Phosphate permeases of Saccharomyces cerevisiae: structure, function and regulation. Biochim Biophys Acta 1999; 1422:255-272.

46 Versaw WK, Metzenberg RL. Repressible cation-phosphate symporters in Neurospora crassa. Proc Natl Acad Sci USA 1995; 92:3884-3887.

47 Gill HS, Pfluegl GM, Eisenberg D. Multicopy crystallographic refinement of a relaxed glutamine synthetase from Mycobacterium tuberculosis highlights flexible loops in the enzymatic mechanism and its regulation. Biochemistry 2002; 41:9863-9872.

48 Liaw SH, Kuo I, Eisenberg D. Discovery of the ammonium substrate site on glutamine synthetase, a third cation binding site. Protein Sci 1995; 4:2358-2365.

49 Thongboonkerd V, Chiangjong W, Saetun P, et al. Analysis of differential proteomes in pathogenic and non-pathogenic Leptospira: potential pathogenic and virulence factors. Proteomics 2009; 9:3522-3534.

50 Buist G, Steen A, Kok J, Kuipers OP. LysM, a widely distributed protein motif for binding to (peptido)glycans. Mol Microbiol 2008; 68:838-847.

51 Kajimura J, Fujiwara T, Yamada S, et al. Identification and 
molecular characterization of an N-acetylmuramyl-L-alanine amidase Sle1 involved in cell separation of Staphylococcus aureus. Mol Microbiol 2005; 58:1087-1101.

52 Hogan RJ, Mathews SA, Kutlin A, Hammerschlag MR, Timms P. Differential expression of genes encoding membrane proteins between acute and continuous Chlamydia pneumoniae infections. Microb Pathog 2003; 34:11-16.

53 Lowder BJ, Duyvesteyn MD, Blair DF. FliG subunit arrangement in the flagellar rotor probed by targeted cross-linking. $J$ Bacteriol 2005; 187:5640-5647.

54 Brown PN, Hill CP, Blair DF. Crystal structure of the middle and $\mathrm{C}$-terminal domains of the flagellar rotor protein FliG. EMBO J 2002; 21:3225-3234.

55 Jacobson EL, Cervantes-Laurean D, Jacobson MK. Glycation of proteins by ADP-ribose. Mol Cell Biochem 1994; 138:207212.

56 Okuda K, Hayashi H, Nishiyama Y. Systematic characterization of the ADP-ribose pyrophosphatase family in the Cyanobacterium Synechocystis sp. strain PCC 6803. J Bacteriol 2005; 187:4984-4991.

57 Kang LW, Gabelli SB, Cunningham JE, O'Handley SF, Amzel LM. Structure and mechanism of MT-ADPRase, a nudix hydrolase from Mycobacterium tuberculosis. Structure 2003; 11:1015-1023.

58 Hinnerwisch J, Fenton WA, Furtak KJ, Farr GW, Horwich AL. Loops in the central channel of ClpA chaperone mediate protein binding, unfolding, and translocation. Cell 2005; 121:1029-1041.

59 Guo F, Maurizi MR, Esser L, Xia D. Crystal structure of ClpA, an Hsp100 chaperone and regulator of ClpAP protease. J Biol Chem 2002; 277:46743-46752.

60 Ansong C, Purvine SO, Adkins JN, Lipton MS, Smith RD. Proteogenomics: needs and roles to be filled by proteomics in genome annotation. Brief Funct Genomic Proteomic 2008; 7:50-62.

61 Tanner S, Shen Z, Ng J, et al. Improving gene annotation using peptide mass spectrometry. Genome Res 2007; 17:231239.

62 Wang R, Prince JT, Marcotte EM. Mass spectrometry of the M. smegmatis proteome: protein expression levels correlate with function, operons, and codon bias. Genome Res 2005; 15:1118-1126.

63 Vieira ML, Pimenta DC, de Morais ZM, Vasconcellos SA, Nascimento AL. Proteome analysis of Leptospira interrogans virulent strain. Open Microbiol J 2009; 3:69-74.

64 Nascimento AL, Verjovski-Almeida S, Van Sluys MA, et al. Genome features of Leptospira interrogans serovar Copenhageni. Braz J Med Biol Res 2004; 37:459-477.

65 Louvel H, Bommezzadri S, Zidane $\mathrm{N}$, et al. Comparative and functional genomic analyses of iron transport and regulation in Leptospira spp. J Bacteriol 2006; 188:7893-7904.

66 Matsunaga J, Barocchi MA, Croda J, et al. Pathogenic Leptospira species express surface-exposed proteins belonging to the bacterial immunoglobulin superfamily. Mol Microbiol 2003; 49:929-945.
67 Lin YP, Raman R, Sharma Y, Chang YF. Calcium binds to leptospiral immunoglobulin-like protein, LigB, and modulates fibronectin binding. $J$ Biol Chem 2008; 283:25140-25149.

68 Croda J, Figueira CP, Wunder EA Jr, et al. Targeted mutagenesis in pathogenic Leptospira species: disruption of the LigB gene does not affect virulence in animal models of leptospirosis. Infect Immun 2008; 76:5826-5833.

69 Haake DA, Matsunaga J. Leptospira: a spirochaete with a hybrid outer membrane. Mol Microbiol 2010; 77:805-814.

70 Ewing B, Hillier L, Wendl MC, Green P. Base-calling of automated sequencer traces using phred. I. Accuracy assessment. Genome Res 1998; 8:175-185.

71 Gordon D, Abajian C, Green P. Consed: a graphical tool for sequence finishing. Genome Res 1998; 8:195-202.

72 Delcher AL, Harmon D, Kasif S, White O, Salzberg SL. Improved microbial gene identification with GLIMMER. Nucleic Acids Res 1999; 27:4636-4641.

73 Lukashin AV, Borodovsky M. GeneMark.hmm: new solutions for gene finding. Nucleic Acids Res 1998; 26:1107-1115.

74 Marchler-Bauer A, Anderson JB, Cherukuri PF, et al. CDD: a Conserved Domain Database for protein classification. Nucleic Acids Res 2005; 33:D192-196.

75 Lowe TM, Eddy SR. tRNAscan-SE: a program for improved detection of transfer RNA genes in genomic sequence. Nucleic Acids Res 1997; 25:955-964.

76 Carver TJ, Rutherford KM, Berriman M, et al. ACT: the artemis comparison tool. Bioinformatics 2005; 21:3422-3423.

77 Tamura K, Dudley J, Nei M, Kumar S. MEGA4: molecular evolutionary genetics analysis (MEGA) software version 4.0. Mol Biol Evol 2007; 24:1596-1599.

78 Kumar A, Tyagi NK, Arevalo E, Miller KW, Kinne RK. A proteomic study of sodium/D-glucose cotransporter 1 (SGLT1): topology of loop 13 and coverage of other functionally important domains. Biochim Biophys Acta 2007; 1774:968-974.

79 Peng J, Elias JE, Thoreen CC, Licklider LJ, Gygi SP. Evaluation of multidimensional chromatography coupled with tandem mass spectrometry (LC/LC-MS/MS) for large-scale protein analysis: the yeast proteome. J Proteome Res 2003; 2:43-50.

80 Baerenfaller K, Grossmann J, Grobei MA, et al. Genomescale proteomics reveals Arabidopsis thaliana gene models and proteome dynamics. Science 2008; 320:938-941.

81 Mallick P, Schirle M, Chen SS, et al. Computational prediction of proteotypic peptides for quantitative proteomics. Nat Biotechnol 2007; 25:125-131.

82 Kal AJ, van Zonneveld AJ, Benes V, et al. Dynamics of gene expression revealed by comparison of serial analysis of gene expression transcript profiles from yeast grown on two different carbon sources. Mol Biol Cell 1999; 10:1859-1872.

83 Lu P, Vogel C, Wang R, Yao X, Marcotte EM. Absolute protein expression profiling estimates the relative contributions of transcriptional and translational regulation. Nat Biotechnol 2007; 25:117-124.

(Supplementary information is linked to the online version of the paper on the Cell Research website) 